\title{
Diversidad florística de la cuenca alta del río Tambo-Ichuña (Moquegua, Perú)
}

\section{Floristic diversity of the upper river basin Tambo-Ichuña (Moquegua, Peru)}

\author{
Daniel B. Montesinos-Tubée
}

NCP Group, Wageningen University. Netherlands. Steinerbos 229 2134JX Hoofddorp, Netherlands. Direción actual: Calle 110 , Direccion actus: Calle 125, pan Mén pa, Peú. dhmtperu@gmail.con damontesinos@yahoo.com

\begin{abstract}
Resumen
La diversidad florística de plantas vasculares es estudiada en la cuenca del río Tambo-lchuña, la puna y bofedales altoandinos en los distritos de Ichuña, Ubinas y Yunga (3400 - $4700 \mathrm{~m}$ de altitud), provincia General Sánchez Cerro, departamento de Moquegua, Perú. La flora vascular de esta región está integrada por 70 familias, 238 géneros y 404 especies. Las Magnoliopsida representan el $78 \%$ de las especies, las Liliopsida $16 \%$, Pteridófitos $6 \%$ y Gimnospermas $0,5 \%$. Se han identificado diez formas (biológicas) de vida, siendo los hemicriptófitos las más numerosas; y tres formaciones vegetales, constituyendo el matorral subhúmedo el más diverso. Entre especies endémicas, 42 taxones son exclusivos para Perú. Son adicionados 272 especies a la flora del departamento de Moquegua.
\end{abstract}

Palabras Clave: flora vascular, Moquegua, formaciones vegetales, formas de vida, especies endémicas

\section{Abstract}

A study of the floristic diversity of vascular plants is presented from the basin of the Tambo-Ichuña River, the high Andean Puna plateau and wetlands of Ichuña, Ubinas and Yunga Districts (3400 - 4700 m altitude), General Sanchez Cerro Province, Department of Moquegua, Peru. Vascular flora is composed of 70 families, 238 genera and 404 species. The Magnoliopsida represent $78 \%$ of the species, Liliopsida $16 \%$, Gymnosperms $0.5 \%$ and Pteridophytes $6 \%$. Among lifeforms, the Hemicryptophytes are the most numerous. Three vegetation formations have been identified, the humid scrubland being the most diverse in species richness. Between endemic species, 42 taxa are exclusive to Peru. A total number of 272 new additions of vascular species to the flora of the department of Moquegua are presented.

Keywords: vascular flora, Moquegua, vegetation formations, lifeforms, endemic species.

\section{Introducción}

El área de estudio se ubica en la provincia General Sánchez Cerro, al norte del departamento de Moquegua, sur del Perú e incluye la cuenca hidrográfica del río Tambo-Ichuña; es un área de difícil acceso y duras condiciones climáticas, en ella se encuentran una variedad de pequeńos ecosistemas andinoaltiplánicos que albergan especies de flora y fauna de interés para la conservación de la biodiversidad. La biodiversidad de esta área se encuentra amenazada por la reforestación con especies exóticas como Eucalyptus rostrata (conocida por degradar suelos, Doughty 2000) y Pinus insignis.

Pocos trabajos botánicos sobre el departamento de Moquegua han sido publicados (Ferreyra 1961, Arakaki \& Cano 2003; Gálan de Mera et al. 2003; Schwarzer et al. 2010). Material de herbario procedente de Moquegua es parte de las colecciones realizadas por A. Weberbauer, R. Ferreyra, M. Dillon, M. Arakaki, M. Weigend, F. Cáceres, P. Peterson y M. Blanchard, sin embargo, material de la zona estudiada en este trabajo es muy escaso.

De acuerdo con el Plan Director de estrategias del sistema Nacional de Áreas Protegidas del Perú (INRENA 1995), la cordillera meridional de los Andes occidentales, que incluye parte de los departamentos de Arequipa, Moquegua y Tacna, es un área con vacio de información acerca de su diversidad biológica (Rodríguez 1996).

El presente trabajo proporciona información recolectada durante los años 2005, 2006 y 2009 sobre la riqueza florística de las quebradas que desembocan en la cuenca del río Tambo-Ichuña en parte de la zona altiplánica, lagunas y bofedales ubicados en la provincia General Sánchez Cerro.

\section{Área de estudio}

El área de estudio comprende la cuenca alta de los ríos TamboIchuña, en la provincia General Sánchez Cerro, Moquegua. Se seleccionaron un total de 56 sectores distribuidos en 12 poblados en los distritos de Ubinas, Yunga y Ichuña, en una gradiente altitudinal de $3400-4700 \mathrm{~m}$ (Tabla 1, Fig. 1). Las colectas
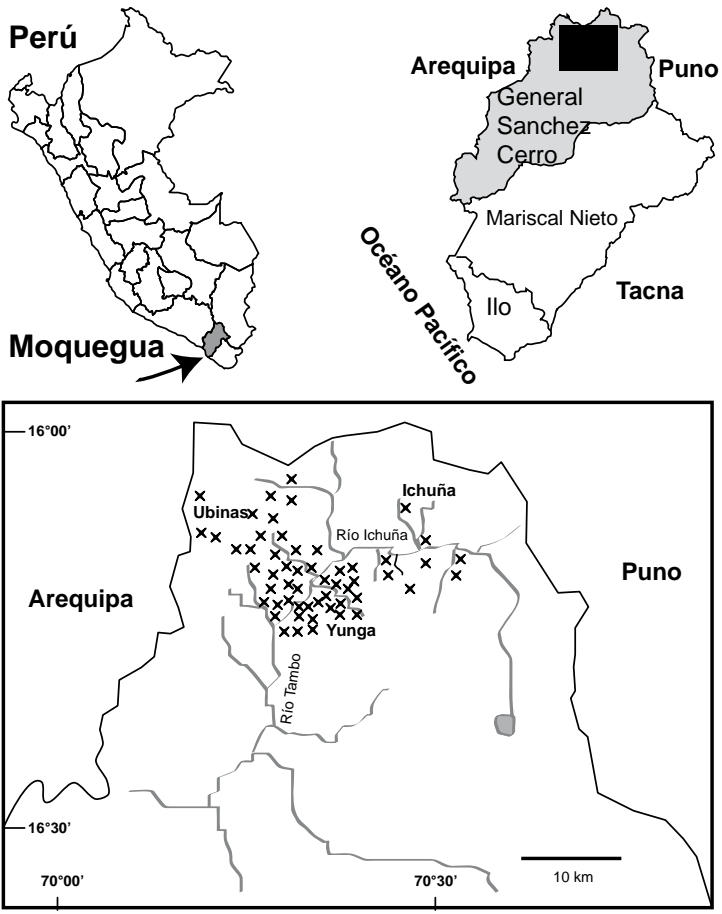

Figura 1. Cuenca de los ríos Tambo-Ichuña en el norte del Departamento de Moquegua indicando la ubicación del área de estudio, los distritos y localidades de muestreo $(\mathrm{x})$. 
Tabla 1. Distritos y respectivas áreas de colecta en el departamento de Moquegua.

\begin{tabular}{ccccc}
\hline Distrito Localidad & $\begin{array}{c}\text { Altura } \\
(\mathbf{m})\end{array}$ & Coordenadas Geográficas & $\begin{array}{c}\text { \# Áreas } \\
\text { de colecta }\end{array}$ \\
\hline Ichuña Ichuña & 3810 & $16^{\circ} 09^{\prime} 26^{\prime \prime} \mathrm{S}$ & $70^{\circ} 39^{\prime} 43^{\prime \prime} \mathrm{W}$ & 4 \\
Italpallune & 3850 & $16^{\circ} 08^{\prime} 33^{\prime \prime} \mathrm{S}$ & $70^{\circ} 29^{\prime} 20^{\prime \prime} \mathrm{W}$ & 2 \\
$\begin{array}{c}\text { Santa Cruz } \\
\text { de Oyo Oyo }\end{array}$ & 3790 & $16^{\circ} 08^{\prime} 27^{\prime \prime} \mathrm{S}$ & $70^{\circ} 32^{\prime} 10^{\prime \prime} \mathrm{W}$ & 2 \\
\hline Sefincane & 3576 & $16^{\circ} 10^{\prime} 10^{\prime \prime} \mathrm{S}$ & $70^{\circ} 39^{\prime} 45^{\prime \prime} \mathrm{W}$ & 1 \\
Ubinas Camata & 3600 & $16^{\circ} 12^{\prime} 50^{\prime \prime} \mathrm{S}$ & $70^{\circ} 42^{\prime} 52^{\prime \prime} \mathrm{W}$ & 8 \\
Coalaque & 4250 & $16^{\circ} 05^{\prime} 44^{\prime \prime} \mathrm{S}$ & $70^{\circ} 43^{\prime} 51^{\prime \prime} \mathrm{W}$ & 7 \\
Querala & 4474 & $16^{\circ} 08^{\prime} 04^{\prime \prime} \mathrm{S}$ & $70^{\circ} 45^{\prime} 19^{\prime \prime} \mathrm{W}$ & 2 \\
Tassa & 3800 & $16^{\circ} 10^{\prime} 54^{\prime \prime} \mathrm{S}$ & $70^{\circ} 41^{\prime} 51^{\prime \prime} \mathrm{W}$ & 10 \\
\hline Yunga Cota & 3586 & $16^{\circ} 10^{\prime} 16^{\prime \prime} \mathrm{S}$ & $70^{\circ} 39^{\prime} 40^{\prime \prime} \mathrm{W}$ & 2 \\
Exchaje & 3480 & $16^{\circ} 13^{\prime} 46^{\prime \prime} \mathrm{S}$ & $70^{\circ} 43^{\prime} 35^{\prime \prime} \mathrm{W}$ & 4 \\
Pampilla & 3540 & $16^{\circ} 12^{\prime} 35^{\prime \prime} \mathrm{S}$ & $70^{\circ} 41^{\prime} 50^{\prime \prime} \mathrm{W}$ & 7 \\
Yunga & 3630 & $16^{\circ} 11^{\prime} 43^{\prime \prime} \mathrm{S}$ & $70^{\circ} 49^{\prime} 39^{\prime \prime} \mathrm{W}$ & 7 \\
\hline
\end{tabular}

botánicas se llevaron a cabo durante los meses de setiembre 2005 y julio 2006, marzo y mayo de 2009.

1. Distrito Ubinas, situado en el flanco derecho del río Tambo y río Paltuture, limitando al oeste con Arequipa, al norte con Puno y al sur con el distrito de Matalaque. Por este distrito atraviesa parte de la antigua carretera Arequipa-Puno; el paisaje presenta quebradas y en el altiplano pajonales, bofedales y lagunas. El estudio se realizó en las laderas y quebradas del río Tambo que bordean los poblados de Camata y Tassa y la puna entre las localidades de Querala y Coalaque. Se seleccionaron 27 sectores en una gradiente altitudinal de $3400-4700 \mathrm{~m}$.

2. Distrito Yunga, ubicado en el flanco izquierdo del río Tambo, limitando al norte con los distritos de Ubinas y Ichuña, al sur con el distrito de Lloque, al este Ichuña y Lloque y al oeste el distrito de Ubinas. Se estudiaron un total de 20 sectores seleccionados entre laderas, quebradas y pajonales en zonas aledañas a los poblados de Yunga, Pampilla y Exchaje, entre 3400 y $4200 \mathrm{~m}$

3. Distrito Ichuńa, comprende en su mayoría localidades altiplánicas, limitando al norte y este con el departamento de Puno, al oeste con los distritos de Ubinas, Yunga y Lloque, y al sur con el distrito de Chojata. Se designaron 9 sectores ubicados entre los poblados de Italpallune, Ichuña y Santa Cruz de Oyo Oyo, en una gradiente altitudinal de $3800-4500 \mathrm{~m}$. El distrito de Ichuña se encuentra entre los más alejados y menos estudiados, resaltando por su gran dimensión territorial y variedad de ecosistemas altiplánicos de suelos crioturbados.

En Ubinas (3200 m) la temperatura media anual oscila entre los $9-11^{\circ} \mathrm{C}$, con una precipitación media anual de $300-360$ $\mathrm{mm}$, en Ichuña $(3800 \mathrm{~m}$ ) la precipitación alcanza los 400 - 500 $\mathrm{mm}$ /año. El clima está caracterizado por ser seco y frío, con un period lluvioso variable que se inicia en noviembre extendiéndose hasta abril.

Los suelos en la cuenca de los ríos Tambo e Ichuña son similares a otras áreas del sur de Perú, constituidas por suelos arcillolimosos en los flancos de las quebradas y en la parte altiplánica arenosos de origen volcánico y crioturbados.

\section{Materiales y métodos}

\section{Diversidad florística}

El material botánico fue recolectado según el método convencional (Maden 2004), además, se tomaron datos de la ubicación geográfica, altitud y estado de conservación de las diferentes especies.

Las determinaciones botánicas se realizaron en el Herbarium Arequipense (HUSA, Arequipa) durante el 2005 y 2006, Herbario San Marcos (USM, Lima) en el 2006 y 2009, Herbario Weberbauer (MOL, Lima) 2006 y 2009, Herbario Universidad Nacional de Cajamarca (CPUN, Cajamarca) 2006, Missouri Botanical Garden (MO, Saint Louis) 2009, National Herbarium Nederland (L, Leiden; WAG, Wageningen) 2007, 2008 y 2010. Se utilizó literatura especializada, claves taxonómicas y la ayuda de especialistas botánicos en la determinación de ejemplares.

Las colectas de campo fueron posteriormente divididas en duplicados con sus etiquetas correspondientes y entregadas a los herbarios HUSA, USM, CPUN, CUZ, HUPCH, MO, WAG [a consolidarse en L] y a la FCBQ de la Universidad Católica de Santa María de Arequipa. La sistemática de las familias está de acuerdo con Angiosperm Phylogeny Group (2009).

Formaciones vegetales.- En el presente estudio se identificaron tres formaciones vegetales (Weberbauer 1942, Huber \& Riina 1997):

Piso mesotérmico de Tolares o Matorral subhúmedo (ms), situado en la vertiente y quebradas de los ríos Tambo-Ichuña $(3400-4200 \mathrm{~m})$, caracterizado por árboles de bajo tamaño (Buddleja coriacea Remy, Escallonia myrtilloides L. f., Polylepis besserii Hieron., Ribes brachybothrys (Wedd.) Jancz.), diversos arbustos, suculentas en áreas rocosas, pastos, hierbas anuales y algunas especies introducidas en las partes cercanas a los cultivos y oconales, este matorral es relativamente ralo, con arbustos que alcanzan 1,5 m de alto, constituidos principalmente por Asteráceas y numerosas anuales.

Pajonal (pj), ubicado entre 4200 y 4700 m, con vegetación altoandina, con predominancia de arbustos bajos, rastreros y hasta pulvinados, gramíneas, entre otras perennes y hierbas anuales. En los flancos de algunas vertientes predominan los pastizales inundados donde desarrollan Juncáceas, Poáceas, Asteráceas y Gentianáceas.

Bofedales (bf), localizados entre 4500 y $4700 \mathrm{~m}$ en suelos saturados de agua, con especies pulviniformes o porte almohadillado (Apiaceae, Asteraceae, Juncaceae, Poaceae) y pequeñas especies de porte arrosetado como Nototriche (Malvaceae), Hypochaeris (Asteraceae), entre otras. Las lagunas permanentes y ocasionales situadas entre los 4400 y 4700 m son también características y hábitat de especies acuáticas.

\section{Formas de vida}

Las formas (biológicas) de vida (Cabrera 1968) se determinaron para todas las especies. Siguiendo el sistema de Raunkiaer (Ellenberg \& Mueller-Dombois 1967) las formas de vida de la zona de estudio se clasificaron en:

Fanerófitos. Arboles pequeños, destacan: Buddleja, Escallonia, Polylepis. 
Tabla 2. Diversidad de familias, géneros y especies registradas en el presente estudio.

\begin{tabular}{lccc}
\hline & Familias & Géneros & Especies* $^{*}$ \\
\hline Pteridophytas & 10 & 17 & 23 \\
Gimnospermas & 1 & 1 & 2 \\
Monocots & 12 & 45 & 65 \\
Eudicots & 47 & 175 & 314 \\
Total & 70 & 238 & 404 \\
\hline
\end{tabular}

Nanofanerófitos. Arbustos con yemas de renuevo a mas de 25 $\mathrm{cm}$ de altura, incluyendo, arbustos de hojas escamiformes (Parastrephia), espiniformes (Chuquiraga), dimorfas (Junellia) y arbustos áfilos (Ephedra).

Caméfitos. Plantas leñosas o sufruticosas con yemas de renuevo pocos centimetros por encima del suelo, incluyendo, sufrútices (Lupinus), caméfitos pulvinados (Azorella, Pycnophyllum), en placas (Astragalus punensis), arbustos rastreros (Stevia mandonii, Salpichroa).

Hemicriptófitos. Plantas herbáceas perennes con yemas de renuevo al ras del suelo, incluyendo, graminiformes (Poaceas y Juncaceas), arrosetados (Hypochaeris, Plantago), rastreros (Sarcostemma, Heliotropium), erectos (Astragalus, Nicotiana).

Helófitos. Con yemas de renuevo bajo un suelo húmedo a inundado (Thelypteris, Distichia, Plantago).

Hidrófitos. Con yemas de renuevo en el agua. Lilaeopsis, Plagiobothrys kunthii (Wal.p) I.M. Johnst. (nuevo reporte para Perú), Isoëtes, Zannichellia.

Fanerófitos suculentos. Plantas crasas, generalmente sin hojas.

Geófitos. Con yemas de renuevo sobre órganos subterráneos, incluyendo, tuberiferos (Ipomoea minuta, Solanum) y bulbíferos (Sisyrinchium, Nothoscordum).

Terófitos, plantas anuales de ciclo vegetativo corto.

\section{Análisis de similitud}

Para el análisis de similitud fueron considerados los siguientes listados florísticos: cuenca río Ilo-Moquegua (Arakaki \& Cano 2003), Reserva Nacional de Nacional Salinas y Aguada Blanca (INRENA 2001), subcuenca río Cotahuasi (L. Velarde, Arequipa, comunicación personal), vegetación altoandina del oeste de Bolivia (Pestalozzi et al. 1998) y vegetación de Tacna (Franco et al. 2004). Las comparaciones se realizaron obteniendo el indice de Sørensen (Mueller-Dombois \& Ellenberg, 1974):

$$
\text { Ss }=2 \mathrm{c} /(\mathrm{a}+\mathrm{b})
$$

Donde: $\mathrm{a}=$ número de especies en el listado $\mathrm{A}$

$\mathrm{b}=$ número de especies en el listado $\mathrm{B}$

$\mathrm{c}=$ número de especies en comunes en $\mathrm{A}$ y $\mathrm{B}$.

Otros listados florísticos fueron excluidos del análisis ya que el reducido número de especies presentes en relación con las otras localidades podría afectar las comparaciones realizadas mediante el análisis de similitud. Las especies descritas a menos de 3000 $\mathrm{m}$ de altitud se omitieron del análisis.

\section{Resultados}

Diversidad Florística.- Se registraron un total de 404 especies, agrupadas en 238 géneros y 70 familias (Apéndice 1). Las
Tabla 3. Familias con mayor riqueza de géneros y especies en la cuenca del río Tambo-Ichuña, Moquegua.

\begin{tabular}{lcc}
\hline Familia & Géneros & Especies \\
\hline Asteraceae & 45 & 97 \\
Poaceae & 22 & 33 \\
Brassicaceae & 13 & 19 \\
Fabaceae & 9 & 18 \\
Solanaceae & 5 & 14 \\
Malvaceae & 3 & 14 \\
Cactaceae & 8 & 13 \\
Caryophyllaceae & 9 & 12 \\
Plantaginaceae & 2 & 12 \\
Apiaceae & 8 & 10 \\
Scrophulariaceae & 6 & 9 \\
Amaranthaceae & 6 & 8 \\
Juncaceae & 4 & 8 \\
Pteridaceae & 4 & 8 \\
Lamiaceae & 4 & 6 \\
Valerianaceae & 2 & 6 \\
Cyperaceae & 5 & 5 \\
Boraginaceae & 4 & 5 \\
OTROS & 79 & 106 \\
\hline
\end{tabular}

Eudicots representan el 78\% de los taxones con 313 especies, las Monocots el $16 \%$, las Gnetophyta representadas por dos especies $(0,5 \%)$ y los Pteridófitos con 23 especies (5,8\%) (Tabla 2).

Las familias más representativas (Tabla 3) son Asteraceae (24\%), Poaceae (5,5\%), Brassicaceae (4,75\%), Fabaceae (4,5\%), Cactaceae (3,25\%), Caryophyllaceae (3\%), Malvaceae (3,5\%), Solanaceae (3,5\%), Plantaginaceae (3\%), Apiaceae (2,5\%) y Scrophulariaceae (2,25\%). Las familias restantes están representadas por menos de 9 especies (37,75\% del total).

En cuanto al hábito, las herbáceas alcanzan valores de $62,5 \%$ siendo la mayoría estacionales, apareciendo durante el inicio de la temporada de lluvias y destacando en todos los tipos de vegetación. Las formas arbustivas $(14,1 \%)$ son en su mayoría caducifolias salvo algunas (Baccharis spp., Parastrephia spp.), adicionalmente, subarbustivas $8,3 \%$, tallos suculentos $3,4 \%$, arbóreas $0,8 \%$, epífitas $0,4 \%$ y parásitas $0,4 \%$.

Se documentan 272 nuevos registros para el departamento de Moquegua que incluyen exclusivamente las colectas identificadas al nivel de especies. Para Pteridófitos se reportan 20 nuevos registros, Monocots con 43 especies y Eudicots con 209 especies de las cuales Asteraceae (68 especies), Poaceae (19 especies), Malvaceae (13 especies) y Brassicaceae con 12 especies, reportan el mayor número de nuevos registros, entre otras familias.

Formaciones vegetales $\mathbf{y}$ formas de vida.- Las formaciones vegetales (Weberbauer 1945; Huber \& Riina, 1997) reflejan la riqueza florística altoandina, habiéndose identificado: el matorral subhúmedo (ms) ubicado entre 3400 y $4200 \mathrm{~m}$ representando el $68,4 \%$ del total de taxones registrados, mientras que los pajonales (pj) en la gradiente altitudinal de $4200-4700 \mathrm{~m}$, registran el 47,8\% de taxones; los bofedales y lagunas (bf, 4400 $-4700 \mathrm{~m}) 11 \%$ respectivamente. Diversos taxones se encuentran presentes y con variada abundancia en la gradiente altitudinal de 3400 - 4700 m, donde destacan: Baccharis tricuneata (L. f.) Pers., Belloa piptolepis (Wedd.) Cabrera, Chersodoma jodopappa 


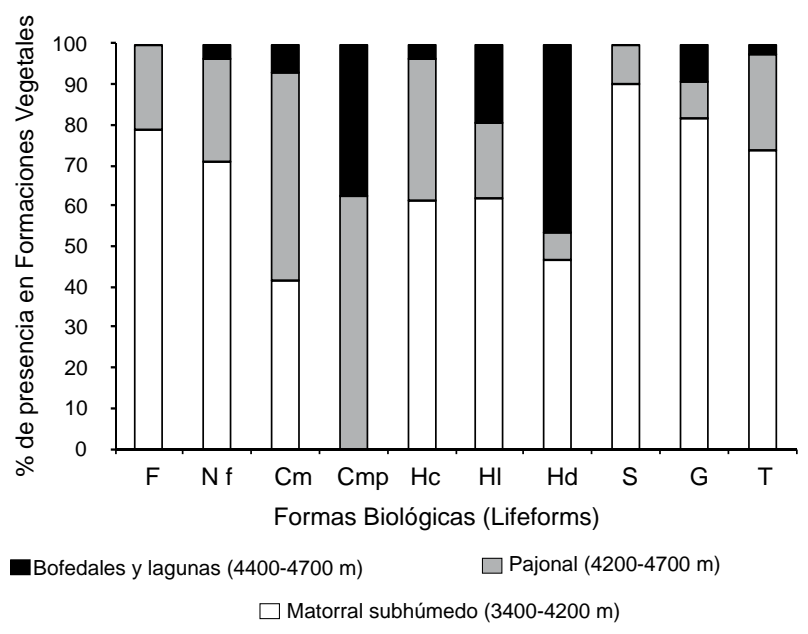

Figura 2. Distribución porcentual de las formas (biológicas) de vida respecto a las formaciones vegetales, en donde: Fanerófito $(F)$; Nanofanerófito (Nf); Caméfitos (Cm); Hemicriptófitos (Hc); Helófitos (HI); Hidrófitos (Hd); Fanerófitos Suculentos (S); Geófitos (G) y Terófitos (T).

(Sch. Bip.) Cabrera, Crassula connata (R. \& P.) Berger., Nassella inconspicua Presl., Parastrephia lepidophylla (Wedd.) Cabrera, Poa candamoana Pilger, Stipa ichu (R. \& P.) Kunth.

Las formas (biológicas) de vida (Fig. 2) incluye en su mayoría Hemicriptófitas (40,8\%), Halófitas (12,6\%), Nanofanerófitas (11,9\%), Caméfitas (5,6\%), Geófitas (3,8\%), fanerófitos suculentos $(3,8 \%)$, Hidrófitas $(3,1 \%)$ y Fanerófitas (2,2\%). Las terófitas constituyen el $16,1 \%$ del total, en tanto las hemicriptófitas $(40,8 \%)$ que incluye la mayoría de gramíneas y especies nativas, predominan en la cuenca alta del rio Tambo-Ichuña.

Analisis de similitud.- Los resultados del análisis de similitud (Fig. 3, Tabla 4) muestran que las localidades andinas cercanas a la costa y cercanas al altiplano puneńo forman unidades separadas. Todos los valores de similitud entre las localidades aproximadas al altiplano son mayores que los valores entre localidades cercanas a la costa; por ejemplo, la similitud florística entre la sierra de Tacna, cuenca río Cotahuasi, laguna de Salinas y oeste de Bolivia es mayor que la registrada en la cuenca del río Ilo-Moquegua.

En la Tabla 5 se resumen las familias con especies endémicas que suman 42 (10,5\%) para el Perú (León et al. 2006), mientras que el 82,6\% (336 especies) son nativas de los Andes (Brako \& Zarucchi 1993), 6,7\% (27 especies) son introducidas y 5 especies $(1,3 \%)$ cosmopolitas.

\section{Discusión y conclusiones}

En el presente estudio los resultados están basados únicamente en las colecciones y observaciones del autor sin incluir las listas

Tabla 4. Indice de similaridad incluyendo familias, géneros y especies en relación con otros estudios.

\begin{tabular}{cccc}
\hline & Familia & Géneros & Especies \\
\hline Tacna & 0,80 & 0,71 & 0,45 \\
Cotahuasi & 0,80 & 0,60 & 0,42 \\
O Bolivia & 0,78 & 0,64 & 0,38 \\
Salinas & 0,70 & 0,60 & 0,36 \\
Ilo-Moquegua & 0,70 & 0,51 & 0,29 \\
\hline
\end{tabular}

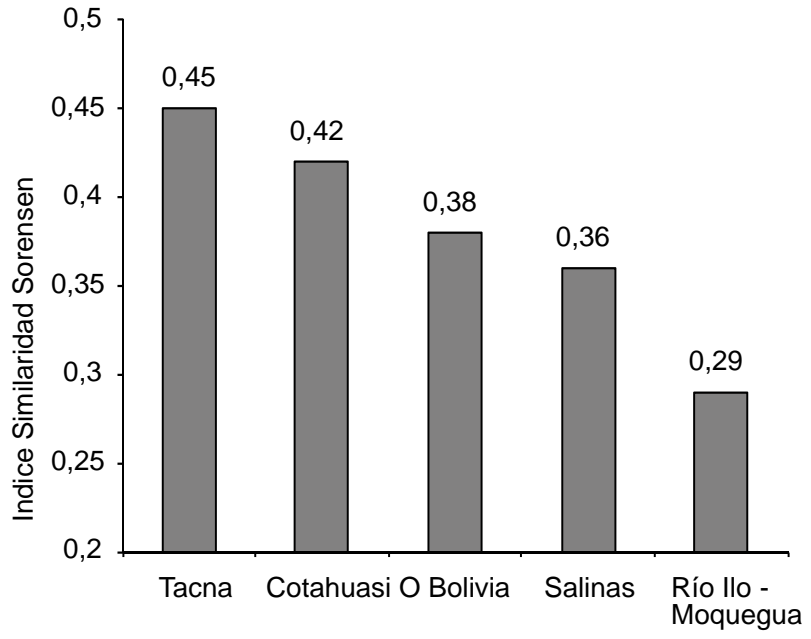

Figura 3. Indice de similaridad de Sorensen comparando el área de estudio con cuatro localidades andinas en Perú y una en Bolivia.

de especies de otros estudios realizados en Moquegua (Arakaki \& Cano 2003, Schwartzer et al. 2010). Los resultados del presente estudio aumentan a 272 nuevos registros botánicos para el departamento de Moquegua.

Diversos autores confirman sobre la riqueza florística en los Andes suroccidentales del Perú (Linares \& Benavides 1995, INRENA 2001, Galán de Mera et al. 2003, Franco et al. 2004, Arteta et al. 2006). Los trabajos de Arakaki \& Cano (2003) y Schwarzer et al. (2010) y Montesinos (2007a) confirman esa tendencia para Moquegua. Asimismo, Montesinos (2007b) afirma sobre la existencia de numerosas especies endémicas en Cactaceae en la cuenca del río Tambo en la sierra del departamento.

Los resultados confirman de una importante contribución de nuevos registros para Moquegua, donde destacan: Asteraceae (68 spp.), Poaceae (19 spp.), Malvaceae (13 spp.), Brassicaceae (12 spp.), Caryophyllaceae, Fabaceae y Plantaginaceae (11 spp. cada una), Juncaceae con 8 especies. En el caso de géneros con mayor número de nuevos registros, se incluye: Senecio con 18

Tabla 5. Número de especies endémicas por familia registradas en la cuenca del río Tambo-Ichuña, Moquegua.

\begin{tabular}{lc}
\hline Familia & \# species endémicas \\
\hline Asteraceae & 12 \\
Cactaceae & 6 \\
Fabaceae & 3 \\
Caryophyllaceae & 2 \\
Crassulaceae & 2 \\
Gentianaceae & 2 \\
Malvaceae & 2 \\
Scrophulariaceae & 2 \\
Valerianaceae & 2 \\
Boraginaceae & 1 \\
Brassicaceae & 1 \\
Calceolariaceae & 1 \\
Geraniaceae & 1 \\
Lamiaceae & 1 \\
Loasaceae & 1 \\
Poaceae & 1 \\
Solanaceae & 1 \\
Verbenaceae & 1 \\
TOTAL & 42 \\
\hline
\end{tabular}


Tabla 6. Géneros con mayor número de especies en la flora de la cuenca del río Tambo-Ichuña.

\begin{tabular}{llc}
\hline Familia & Género & N de especies \\
\hline Asteraceae & Senecio & 20 \\
Plantaginaceae & Plantago & 11 \\
Malvaceae & Nototriche & 9 \\
Solanaceae & Solanum & 8 \\
Fabaceae & Astragalus & 7 \\
Asteraceae & Baccharis & 5 \\
Asteraceae & Werneria & 5 \\
Poaceae & Calamagrostis & 5 \\
Pteridaceae & Cheilanthes & 5 \\
Valerianaceae & Valeriana & 5 \\
Asteraceae & Hypochaeris & 4 \\
Asteraceae & Perezia & 4 \\
Malvaceae & Tarasa & 4 \\
Scrophulariaceae & Bartsia & 4 \\
\hline
\end{tabular}

especies, Plantago con 11 especies, Nototriche con 9 especies, etc. (Tabla 6). Resaltan también el registro de nuevas familias a la flora del departamento: Gentianaceae (3 spp.), Campanulaceae (3 spp.), Aspleniaceae (3 spp.), Violaceae (2 spp.), Orchidaceae (2 spp.), Thelypteridaceae (2 spp.), Callitrichaceae, Grossulariaceae, Lentibulariaceae, Piperaceae, Saxifragaceae con una especie cada una.

También se comprueba que la vegetación de la cuenca TamboIchuña tiene mayor similitud con la del altiplano puneño y boliviano cercano al lago Titicaca y valles altoandinos del NE de Arequipa y Tacna. Dichas regiones forman parte del mismo sistema montańoso subhúmedo del sur de los Andes presentando casi las mismas características altitudinales y de composición florística.

Por otro lado, la vegetación de la cuenca del río TamboIchuña tiene menores afinidades genéricas con lo reportado en la cuenca del río Ilo-Moquegua (Arakaki \& Cano 2003) que tendría mayor influencia de la vegetación de la costa peruana y del norte de Chile (Tabla 4).

La presencia de taxa relacionados con la zona altoandina mas que con la vertiente andino-costera podría ser un indicio que los intercambios florísticos se habrían producido a través de las zonas cordilleranas-altiplánicas de los Andes que estarían actúando como corredores en el desplazamiento norte-sur de la flora vascular, así como se ha sugerido en otros trabajos (Luebert et al. 2009, Pinto \& Luebert 2009, Schwarzer et al. 2010).

Como parte de los resultados se han identificado los rodales de Puya raimondii distribuidas en cinco áreas geográficas en el norte del departamento de Moquegua entre los 3800 y 4200 $\mathrm{m}$ y compuestas por un importante número de ejemplares en diversos estados vegetativos y que requieren ser considerados dentro de los planes de conservación.

\section{Agradecimientos}

Agradezco especialmente a los curadores de los herbarios F, HUSA, HUPCH, L, MO, MOL, USM, WAG por la buena disposición para su consulta. A Francisco Lazo y Jorge Salinas (Universidad Católica de Santa María) por sus valiosos comentarios. A Victor Quipuscoa, Gina Castillo y Fátima Cáceres (Universidad Nacional de San Agustín), Hamilton Beltrán, Blanca León,
Asunción Cano, Magda Chanco, Susy Castillo, Maria Isabel La Torre, Oscar Tovar $(†)$ (Museo de Historia Natural, Lima) por su valiosa colaboración en la determinación de especímenes colectados. Al Dr. Carlos Ostolaza por sus comentarios en Cactaceae. Al Dr. Ihsan Al-Shehbaz (Missouri Botanical Gardens) por sus comentarios en Brassicáceas, A John Pruski y Ronald Liesner (Missouri Botanical Gardens) por su ayuda en determinaciones, a Harvey Ballard (Ohio University) por la identificación de Violáceas. Antoine Cleef (Universiteit van Amsterdam) por sus valiosos comentarios y facilitar bibliografía. Karle Sykora y Jan Wieringa (Wageningen University) por sus sugerencias. A Diego Linares por los mapas y ayuda en los trámites. Luis Mondragón, Edwin Banegas, Christian Pinto, Guillermo Macedo y Lorena Velarde por sus distintas contribuciones en las diferentes etapas del desarrollo la investigación. A dos revisores anónimos por su importante aporte al contenido y presentación de este trabajo. El autor agradece a las autoridades y población de las diferentes localidades visitadas en los Distritos de Ichuña, Ubinas y Yunga, por compartir sus conocimientos sobre la flora y las facilidades brindadas durante el trabajo de campo. Esta investigación recibió el apoyo financiero del NCP Group (Wageningen University), C. Bünemann, P. Kothe, y S. Montesinos.

El presente trabajo contó con las Autorizaciones 014 -2009-AG-DGFFS-DGEFFS y N_001931-AG-DGFFS- expedidos por la Dirección General Forestal y de Fauna Silvestre. Ministerio de Agricultura, Lima, Perú.

\section{Literatura citada}

Angiosperm Phylogeny Group. 2009. An update of the Angiosperm Phylogeny Group classification for the orders and families of flowering plants: APG III. Botanical Journal of the Linnean Society 161(2): 105-121, Disponible en web: http:// www3.interscience.wiley.com/journal/122630309/abstract (consulta: 1 de Marzo del 2011).

Arakaki M \& A. Cano. 2003. Composición florística de la cuenca del río Ilo-Moquegua y lomas de Ilo, Moquegua. Rev. peru. biol. 10(1): 5-15.

Arteta M., M. Corrales, C. Dávalos, et al. 2006. Plantas Vasculares de la Bahía de Juli, Lago Titicaca, Puno-Perú. Ecología Aplicada, 5(1,2): 1-8.

Brako L. \& J. Zarucchi. 1993. Catálogo de las Angiospermas y Gimnospermas del Perú. Monogr. Syst. Bot. Missouri Bot. Garden. Vol 45.

Cabrera A. 1968. Ecología Vegetal de la Puna. Colloquium Geographicum. Band 9. Proceedings of the UNESCO Mexico Symposium, August 1-3, 1966. Pp. 91-116.

Doughty R. 2000. The Eucalyptus: a natural and commercial history of the gum tree. The Johns Hopkins University Press. Baltimore, MD:p.237.

Ellenberg,H. \& D. Mueller-Dombois. 1967. A key to Raunkiaer life forms with revised subdivitions, Ber. Geobot. Inst. Eidgen. Techn. Hochsch. Stiftung Rübel 37: 56-73.

Ferreyra R. 1961. Las lomas costaneras del extremo sur del Perú. Boletín de la Sociedad Argentina de Botánica, 9: 85-100.

Franco J., C. Cáceres \& J. Sulca. 2004. Flora y Vegetación del departamento de Tacna, Perú. Ciencia y Desarrollo 8: 23-30.

Galán de Mera, A., C. Cáceres \& A. Gonzáles. 2003. La vegetación de la alta montaña andina del sur de Perú. Acta Botánica Malacitana 28: 121-130.

Huber O. \& R. Riina, 1997. Glosario fitoecológico de las Américas. Vol. 1. América del Sur: países hispanohablantes. UNESCO y Fundación Instituto Botánico de Venezuela. Caracas. Pp. 416-421. 
INRENA (Instituto Nacional de Recursos Naturales). 1995. Estrategia del Sistema Nacional de Áreas Naturales Protegidas del Perú. Plan Director. Proyecto FANPE. BIB / 2540. Pp 1-95.

INRENA (Instituto Nacional de Recursos Naturales). 2001. Plan Maestro de la Reserva Nacional Salinas y Aguada Blanca. Resolución Jefatural No 136-2001-INRENA. Ministerio de Agricultura, Lima.

León B. 1993. Catálogo anotado de las fanerógamas acuáticas del Perú. En: F. Kahn, B. León \& K.R. Young (eds.). Las Plantas Vasculares en las Aguas Continentales del Perú. Tomo 75, Travaux de l'Institut Français d'Études Andines. (IFEA). Lima. Pp. 110-128.

León B., J. Roque, C. Ulloa Ulloa, et al. 2006. Libro Rojo de las Plantas endémicas del Perú. Revista Peruana de Biología, Edición Especial 13(2): 971 pp.

Linares E. \& M. Benavides. 1995. Flora silvestre del transecto Yura-Chivay, departamento de Arequipa. Boletín de Lima. 100: 211-254

Luebert F.; J. Wen \& M.O. Dillon. 2009. Systematic placement and biogeographical relationships of the monotypic genera Gypothamnium and Oxyphyllum (Asteraceae: Mutisioideae) from the Atacama Desert. Botanical Journal of the Linnean Society 159: $32-51$

Maden K. 2004. Plant Collection and Herbarium Techniques. Our Nature (2004) 2:53-57
Montesinos D. 2007a. Estudio Botánico y Taxonómico de la Composición Florística en cinco anexos de la Provincia General Sánchez Cerro, Departamento de Moquegua, Perú. Tesis de Bachiller. Programa Profesional de Ingeniería Agronómica, Universidad Católica de Santa María, Arequipa, Perú. Pp. 24-77.

Montesinos D. 2007b. Cactáceas de la Provincia General Sánchez Cerro, Moquegua, Perú. Quepo, 27: 74-87.

Mueller-Dombois, D. \& H. Ellenberg. 1974. Aims and Methods of Vegetation Ecology. University of Minnesota. 312-316 pp.

Pestalozzi H., M. Torrez, 1998. Flora Ilustrada Altoandina. La relación entre hombre, planta y medio ambiente en el Ayllu Majasaya Mujlli. Herbario Forestal Martin Cardenas Cochabamba, Bolivia. Pp. 111-214.

Pinto R. \& F. Luebert. 2009. Datos sobre la flora vascular del desierto costero de Arica y Tarapaca, Chile, y sus relaciones fitogeográficas con el Sur de Peru. Gayana Bot. 66(1): 46.

Rodríguez L. 1996. Diversidad Biológica del Perú, zonas prioritarias para su conservación. Proyecto FAMPE GTZ-INRENA. Ministerio de Agricultura, Instituto Nacional de Recursos Naturales (INRENA), Lima, Perú. Pp. 191

Schwarzer C., F. Cáceres, A. Cano, M.I. La Torre \& M. Weigend. 2010. 400 years for long-distance dispersal and divergence in the northern Atacama Desert and Insights from the Huaynaputina pumice slopes of Moquegua, Peru. Journal of Arid Environments 74(11): 1540-1551

Weberbauer A. 1945. El Mundo Vegetal de los Andes Peruanos. Estación Experimental Agrícola de la Molina. Dirección de Agricultura, Lima, Perú.

Apéndice 1. Lista de la flora vascular para la cuenca del río Tambo-Ichuña (Provincia General Sánchez Cerro, Moquegua, Perú). Son 404 especies, se adicionan 43 taxa identificados a nivel especifico (sp.). FC: forma de crecimiento (h: hierba, hs: subarbusto, s: arbusto, a: árbol, su: suculenta, p: parásita, e: epifita); FV: formación vegetal (ms: matorral subhúmedo, pj: pajonal, bf: bofedal); FB: formas biológicas: Fanerófito $(\mathrm{F})$, Nanofanerófito $(\mathrm{Nf})$, Caméfitos $(\mathrm{Cm})$, Hemicriptófitos $(\mathrm{Hc})$, Helófitos $(\mathrm{HI})$, Hidrófitos $(\mathrm{Hd})$, Fanerófitos suculentos $(\mathrm{S})$, Geófitos $(\mathrm{G})$ y Terófitos (T); St: status de la especie (N: Nativa, E: Endémica, I: Introducida, Co: Cosmopolita); NR: primer registro para Moquegua; Altitud: registro altitudinal de la colección(es); Voucher: Mont. (Daniel B. Montesinos), f!: registro sin material de herbario, solo fotografia, * mas de dos colecciones botánicas.

\begin{tabular}{|c|c|c|c|c|c|c|c|c|}
\hline Taxón & FC & FE & FB & St & NR & Altitud (m) & Voucher & \\
\hline \multicolumn{9}{|l|}{ Pteridophyta } \\
\hline \multicolumn{9}{|l|}{ Adiantaceae } \\
\hline Adiantum subvolubile Mett. ex Kuhn & $\mathrm{h}$ & $\mathrm{ms}$ & $\mathrm{Hl}$ & $\mathrm{N}$ & & $3400-3500$ & Mont. 0626 & \\
\hline \multicolumn{9}{|l|}{ Aspleniaceae } \\
\hline Asplenium gilliesii Hook. & $\mathrm{h}$ & pj & $\mathrm{Hl}$ & $\mathrm{N}$ & $\mathrm{x}$ & $4000-4200$ & Mont. 1177 & \\
\hline Asplenium peruvianum Desv. & $\mathrm{h}$ & $\mathrm{ms}, \mathrm{pj}$ & $\mathrm{Hl}$ & $\mathrm{N}$ & $x$ & $3600-4200$ & Mont. 2250 & * \\
\hline Asplenium triphyllum C. Presl & $\mathrm{h}$ & pj & $\mathrm{Hl}$ & $\mathrm{N}$ & $x$ & 4500 & Mont. 0945 & \\
\hline \multicolumn{9}{|l|}{ Dryopteridaceae } \\
\hline Cystopteris fragilis (L.) Bernh. & $\mathrm{h}$ & $\mathrm{ms}, \mathrm{pj}$ & $\mathrm{Hl}$ & $\mathrm{N}$ & & $3400-3900$ & Mont. 2228 & * \\
\hline Polystichum orbiculatum var. orbiculatum & $\mathrm{h}$ & $\mathrm{ms}, \mathrm{pj}$ & $\mathrm{Hl}$ & $\mathrm{N}$ & $\mathrm{x}$ & $3800-4000$ & Mont. 2034 & * \\
\hline \multicolumn{9}{|l|}{ Equisetaceae } \\
\hline Equisetum bogotense Kunth & $\mathrm{h}$ & $\mathrm{ms}$ & $\mathrm{Hl}$ & $\mathrm{N}$ & & $3400-3700$ & Mont. 0984 & \\
\hline \multicolumn{9}{|l|}{ Isoetaceae } \\
\hline Isoetes sp. & $\mathrm{h}$ & bf & $\mathrm{Hd}$ & - & $x$ & 4400 & Mont. 2588b & \\
\hline \multicolumn{9}{|l|}{ Polypodiaceae } \\
\hline Campyloneurum amphostenon (Kunze ex Klotzsch) Fée & $\mathrm{h}$ & $\mathrm{ms}$ & $\mathrm{Hl}$ & $\mathrm{N}$ & $x$ & 3700 & Mont. 1213 & \\
\hline Elaphoglossum minutum (Pohl ex Fée) T. Moore & $\mathrm{h}$ & ms & $\mathrm{Hl}$ & $\mathrm{N}$ & $x$ & $3500-3600$ & Mont. 2095 & * \\
\hline Melpomene peruviana (Desv.) A.R. Sm. \& R.C. Moran & $\mathrm{h}$ & $\mathrm{ms}$ & $\mathrm{Hl}$ & $\mathrm{N}$ & $\mathrm{x}$ & 3600 & Mont. 1202 & \\
\hline Polypodium pycnocarpum C. Chr. & $\mathrm{h}$ & $\mathrm{ms}$ & $\mathrm{Hl}$ & $\mathrm{N}$ & $x$ & 3500 & Mont. 2339 & * \\
\hline \multicolumn{9}{|l|}{ Pteridaceae } \\
\hline Cheilanthes arequipensis (Maxon) R.M. Tryon \& A.F. Tryon & $\mathrm{h}$ & ms, pj & Hc & $\mathrm{N}$ & $x$ & $3500-4050$ & Mont. 2097 & * \\
\hline Cheilanthes myriophylla Desv. & $\mathrm{h}$ & $\mathrm{ms}$ & Hc & $\mathrm{N}$ & $x$ & $3400-3400$ & Mont. 2218 & * \\
\hline Cheilanthes pilosa Goldm. & $\mathrm{h}$ & pj & Hc & $\mathrm{N}$ & $\mathrm{x}$ & $3700-4200$ & Mont. 2545 & * \\
\hline Cheilanthes pruinata Kaulf. & $\mathrm{h}$ & $\mathrm{ms}, \mathrm{pj}$ & Hc & $\mathrm{N}$ & & $3400-4200$ & Mont. 0514 & \\
\hline Cheilanthes scariosa (Sw.) C. Presl. & $\mathrm{h}$ & $\mathrm{ms}, \mathrm{pj}$ & $\mathrm{Hc}$ & $\mathrm{N}$ & $x$ & $3500-3900$ & Mont. 2244 & * \\
\hline Jamesonia alstonii A. F. Tryon & $\mathrm{h}$ & $\mathrm{ms}$ & $\mathrm{Hl}$ & $\mathrm{N}$ & $\mathrm{x}$ & 3700 & Mont. 2043 & * \\
\hline Notholaena nivea (Poir.) Windham & $\mathrm{h}$ & $\mathrm{ms}$ & Hc & $\mathrm{N}$ & $x$ & $3400-4100$ & Mont. 0510 & \\
\hline Pellaea ternifolia (Cav.) Link & $\mathrm{h}$ & $\mathrm{ms}$ & Hc & $\mathrm{N}$ & $\mathrm{x}$ & $3400-3500$ & Mont. 0543 & * \\
\hline
\end{tabular}




\begin{tabular}{|c|c|c|c|c|c|c|c|c|}
\hline Taxón & FC & FE & FB & St & NR & Altitud (m) & Voucher & \\
\hline \multicolumn{9}{|l|}{ Salviniaceae } \\
\hline Azolla filiculoides Lam. & $\mathrm{h}$ & $\mathrm{ms}, \mathrm{pj}$ & $\mathrm{Hd}$ & I & $\mathrm{x}$ & 3800 & Mont. 2156a & \\
\hline \multicolumn{9}{|l|}{ Thelypteridaceae } \\
\hline Thelypteris glandulosolanosa (C. Chr.) R.M. Tryon & $\mathrm{h}$ & $\mathrm{ms}, \mathrm{pj}$ & $\mathrm{Hd}$ & $\mathrm{N}$ & $\mathrm{x}$ & $3400-3900$ & Mont. 1211 & \\
\hline Thelypteris rufa (Poiret) A.R. Sm. & $\mathrm{h}$ & $\mathrm{ms}$ & $\mathrm{Hl}$ & $\mathrm{N}$ & $\mathrm{x}$ & 3500 & Mont. 0627 & \\
\hline \multicolumn{9}{|l|}{ Woodsiaceae } \\
\hline Woodsia montevidensis (Spreng.) Hieron. & $\mathrm{h}$ & $\mathrm{ms}, \mathrm{pj}$ & $\mathrm{Hl}$ & $\mathrm{N}$ & $\mathrm{x}$ & $3600-4000$ & Mont. 2040 & * \\
\hline \multicolumn{9}{|l|}{ Gnetophyta } \\
\hline \multicolumn{9}{|l|}{ Ephedraceae } \\
\hline Ephedra americana Humb. \& Bonpl. ex Willd. & $\mathrm{s}$ & $\mathrm{ms}$ & $\mathrm{Nf}$ & $\mathrm{N}$ & & $3400-3900$ & Mont. 0658 & \\
\hline Ephedra rupestris Benth. & $\mathrm{s}$ & pj & $\mathrm{Cm}$ & $\mathrm{N}$ & & $4000-4500$ & Mont. 0563 & * \\
\hline \multicolumn{9}{|l|}{ Angiospermas } \\
\hline \multicolumn{9}{|l|}{ Monocots } \\
\hline \multicolumn{9}{|l|}{ Alstroemeriaceae } \\
\hline Alstroemeria pygmaea Herb. & $\mathrm{h}$ & $\mathrm{ms}$ & G & $\mathrm{N}$ & $\mathrm{x}$ & $3800-3900$ & Mont. 2130 & * \\
\hline Bomarea dulcis (Hook) Beauverd & hs & pj & Hc & $\mathrm{N}$ & & $3900-4200$ & Mont. 0637 & * \\
\hline Bomarea involucrosa (Herb.) Baker & $\mathrm{s}$ & $\mathrm{ms}$ & Hc & $\mathrm{N}$ & $\mathrm{x}$ & $3500-4100$ & Mont. 1000 & * \\
\hline Bomarea ovata (Cav.) Mirb. & hs & $\mathrm{ms}$ & Hc & $\mathrm{N}$ & & $3400-3400$ & Mont. 2209 & \\
\hline \multicolumn{9}{|l|}{ Amaryllidaceae } \\
\hline Zephyranthes paroula Killip. & $\mathrm{h}$ & $\mathrm{ms}$ & G & $\mathrm{N}$ & $\mathrm{x}$ & 3400 & $\mathrm{f} !$ & \\
\hline \multicolumn{9}{|l|}{ Asparagaceae } \\
\hline Agave americana $\mathrm{L}$. & s, su & $\mathrm{ms}$ & $\mathrm{F}$ & I & $\mathrm{x}$ & $3400-3700$ & Mont. 1219 & \\
\hline Bromeliaceae & & & & & & & & \\
\hline Puya ferruginea (Ruiz \& Pav.) L.B. Sm. & $\mathrm{s}$ & $\mathrm{ms}$ & $\mathrm{Nf}$ & $\mathrm{N}$ & & $3400-3600$ & Mont. 0623 & \\
\hline Puya raimondii Harms & $\mathrm{s}$ & $\mathrm{ms}, \mathrm{pj}$ & $\mathrm{F}$ & $\mathrm{N}$ & $\mathrm{x}$ & $3800-4200$ & Mont. 2209 & * \\
\hline Tillandsia capillaris Ruiz \& Pav. & $h, e$ & $\mathrm{~ms}$ & Hc & $\mathrm{N}$ & $\mathrm{x}$ & $3400-3900$ & Mont. 0504 & * \\
\hline Tillandsia usneoides (L.) L. & h, e & $\mathrm{ms}$ & $\mathrm{Hc}$ & $\mathrm{N}$ & $\mathrm{x}$ & $3400-3600$ & Mont. 2348 & * \\
\hline Cyperaceae & & & & & & & & \\
\hline Carex cf. collumanthus (Steyerm.) G.A. Wheeler & $\mathrm{h}$ & bf & $\mathrm{Hl}$ & $\mathrm{N}$ & $\mathrm{x}$ & 4600 & $\mathrm{f!}$ & \\
\hline Cyperus seslerioides Kunth & $\mathrm{h}$ & ms, pj & Hc & $\mathrm{N}$ & $\mathrm{x}$ & $3400-4200$ & Mont. 2106 & * \\
\hline Eleocharis albibracteata Nees \& Meyen ex Kunth & $\mathrm{h}$ & $\mathrm{ms}$ & $\mathrm{Hl}$ & $\mathrm{N}$ & & $3500-3700$ & Mont. 2106 & * \\
\hline Isolepis cernua (Vahl) Roem. \& Schult. & $\mathrm{h}$ & $\mathrm{ms}$ & $\mathrm{Hd}$ & $\mathrm{N}$ & $\mathrm{x}$ & 3400 & Mont. 2210 & \\
\hline Isolepis inundata R. Br. & $\mathrm{h}$ & $\mathrm{ms}$ & $\mathrm{Hl}$ & I & $\mathrm{x}$ & 3700 & Mont. 2005 & \\
\hline Rhynchospora sp. & $\mathrm{h}$ & $\mathrm{ms}$ & $\mathrm{Hl}$ & $\mathrm{N}$ & $\mathrm{x}$ & 3400 & Mont. 2223 & \\
\hline Iridaceae & & & & & & & & \\
\hline Cardenanthus sp. & $\mathrm{h}$ & $\mathrm{ms}$ & G & - & - & 3500 & Mont. 2107 & \\
\hline Olsynium junceum (E. Meyer ex Presl) Goldblatt & $\mathrm{h}$ & $\mathrm{ms}$ & G & $\mathrm{N}$ & & $3500-4000$ & Mont. 2153 & * \\
\hline Sisyrinchium bracteosum Phil. & $\mathrm{h}$ & $\mathrm{ms}, \mathrm{pj}$ & G & $\mathrm{N}$ & $\mathrm{x}$ & $3900-4200$ & Mont. 2058 & * \\
\hline Sisyrinchium cf. trinerve Baker & $\mathrm{h}$ & pj & G & $\mathrm{N}$ & $\mathrm{x}$ & 4100 & Mont. 2275 & \\
\hline Sisyrinchium sp. & $\mathrm{h}$ & $\mathrm{bf}$ & G & - & - & 4600 & Mont. 2392 & \\
\hline Juncaceae & & & & & & & & \\
\hline Distichia acicularis Balslev \& Lægaard & $\mathrm{h}$ & bf & $\mathrm{Hl}$ & $\mathrm{N}$ & $\mathrm{x}$ & $3900-4400$ & Mont. 2291 & * \\
\hline Distichia muscoides Nees \& Meyen & $\mathrm{h}$ & $\mathrm{bf}$ & $\mathrm{Hl}$ & $\mathrm{N}$ & $\mathrm{x}$ & $4400-4700$ & Mont. 2378 & * \\
\hline Juncus arcticus var. andicola (Hook.) Balslev & $\mathrm{h}$ & $\mathrm{ms}$ & $\mathrm{Hl}$ & $\mathrm{N}$ & $\mathrm{x}$ & $3400-3500$ & Mont. 1196 & * \\
\hline Juncus cf. stipulatus Nees \& Meyen & $\mathrm{h}$ & pj & $\mathrm{Hl}$ & $\mathrm{N}$ & $\mathrm{x}$ & $4400-4500$ & Mont. 2457b & \\
\hline Juncus ebracteatus E. Mey. & $\mathrm{h}$ & $\mathrm{ms}$ & $\mathrm{Hl}$ & $\mathrm{N}$ & $\mathrm{x}$ & $3400-3700$ & Mont. 2014 & * \\
\hline Luzula racemosa Desv. & $\mathrm{h}$ & $\mathrm{ms}, \mathrm{pj}$ & Hc & $\mathrm{N}$ & $\mathrm{x}$ & $3400-4100$ & Mont. 2220 & * \\
\hline Luzula vulcanica Liebm. & $\mathrm{h}$ & $\mathrm{pj}, \mathrm{bf}$ & Hc & $\mathrm{N}$ & $\mathrm{x}$ & $4200-4500$ & Mont. 2374 & * \\
\hline Oxychloe andina Phil. & $\mathrm{h}$ & $\mathrm{bf}$ & $\mathrm{Hl}$ & $\mathrm{N}$ & $\mathrm{x}$ & $3900-4600$ & Mont. 2291 & \\
\hline Lemnaceae & & & & & & & & \\
\hline Lemna minuscula Herter & $\mathrm{h}$ & bf & $\mathrm{Hl}$ & I & & 4500 & Mont. 0934b & \\
\hline Liliaceae & & & & & & & & \\
\hline Nothoscordum andicola Kunth & $\mathrm{h}$ & $\mathrm{ms}$ & G & $\mathrm{N}$ & & $3400-3600$ & Mont. 0789 & * \\
\hline Nothoscordum fictile J.F. Macbr. & $\mathrm{h}$ & $\mathrm{ms}$ & G & $\mathrm{N}$ & & $3400-3600$ & Mont. 0809 & \\
\hline Orchidaceae & & & & & & & & \\
\hline Aa mathewsii (Rchb. f.) Schltr. & h, su & $\mathrm{ms}$ & G & $\mathrm{N}$ & $\mathrm{x}$ & $3400-3650$ & Mont. 2128 & * \\
\hline Myrosmodes pumilio (Schltr.) C. Vargas & h, su & bf & G & $\mathrm{N}$ & $\mathrm{x}$ & $4050-4650$ & Mont. 2287 & \\
\hline Poaceae & & & & & & & & \\
\hline Aciachne pulvinata Benth. & $\mathrm{h}$ & bf & $\mathrm{Cm}$ & $\mathrm{N}$ & $\mathrm{x}$ & $4600-4700$ & Mont. 2393 & \\
\hline Anatherostipa rigidiseta (Pilg.) Peñailillo & $\mathrm{h}$ & pj & Hc & $\mathrm{N}$ & $\mathrm{x}$ & $4200-4400$ & Mont. 2428b & \\
\hline Aristida adscencionis L. & $\mathrm{h}$ & $\mathrm{ms}, \mathrm{pj}$ & $\mathrm{Hc}$ & $\mathrm{N}$ & & $3500-4300$ & Mont. 2492 & * \\
\hline Bothriochloa saccharoides (Sw.) Rydb. & $\mathrm{h}$ & $\mathrm{ms}$ & $\mathrm{Hc}$ & $\mathrm{N}$ & $\mathrm{x}$ & 3500 & Mont. 0787 & \\
\hline Bromus catharticus Vahl. & $\mathrm{h}$ & $\mathrm{ms}, \mathrm{pj}$ & $\mathrm{Hc}$ & $\mathrm{N}$ & & $3700-4400$ & Mont. 2266 & * \\
\hline Calamagrostis heterophylla (Wedd.) Pilg. & $\mathrm{h}$ & $\mathrm{ms}$ & Hc & $\mathrm{N}$ & & $3500-3700$ & Mont. 2219c & \\
\hline Calamagrostis minima (Pilg.) Tovar & $\mathrm{h}$ & $\mathrm{pj}, \mathrm{bf}$ & Hc & $\mathrm{N}$ & $x$ & $4200-4700$ & Mont. 2469 & * \\
\hline Calamagrostis ovata (J. Presl) Steud. & $\mathrm{h}$ & $\mathrm{bf}$ & $\mathrm{Hl}$ & $\mathrm{N}$ & $\mathrm{x}$ & $4400-4600$ & Mont. 2408 & \\
\hline Calamagrostis rigescens (J. Presl) Scribn. & $\mathrm{h}$ & $\mathrm{pj}, \mathrm{bf}$ & Hc & $\mathrm{N}$ & & $3900-4400$ & Mont. 2066 & * \\
\hline Calamagrostis vicunarum (Wedd.) Pilg. & $\mathrm{h}$ & $\mathrm{ms}, \mathrm{pj}, \mathrm{bf}$ & Hc & $\mathrm{N}$ & $\mathrm{x}$ & $3900-4600$ & Mont. 2435 & * \\
\hline Calamagrostis sp. & $\mathrm{h}$ & $\mathrm{ms}$ & $\mathrm{Hc}$ & - & - & 4450 & Mont. 2483 & \\
\hline
\end{tabular}

(Continúa...) 
Apéndice 1. Continuación.

\begin{tabular}{|c|c|c|c|c|c|c|c|c|}
\hline Taxón & FC & FE & FB & St & NR & Altitud (m) & Voucher & \\
\hline Chondrosum simplex (Lag.) Kunth & $\mathrm{h}$ & $\mathrm{ms}$ & $\mathrm{T}$ & $\mathrm{N}$ & & $3400-3900$ & Mont. 2219a & * \\
\hline Cortaderia bifida Pilg. & $\mathrm{h}$ & $\mathrm{ms}$ & $\mathrm{Hc}$ & $\mathrm{N}$ & $x$ & $3400-3700$ & Mont. 0651 & * \\
\hline Deyeuxia curvula Wedd. & $\mathrm{h}$ & ms, pj & $\mathrm{Hc}$ & $\mathrm{N}$ & & $3900-4200$ & $\mathrm{f} !$ & \\
\hline Dielsiochloa floribunda (Pilg.) Pilg. & $\mathrm{h}$ & $\mathrm{pj}$ & $\mathrm{Hc}$ & $\mathrm{N}$ & & $4400-4600$ & Mont. 2423 & \\
\hline Dissanthelium calycinum (J. Presl) Hitchc. & $\mathrm{h}$ & pj, bf & $\mathrm{T}$ & $\mathrm{N}$ & $x$ & $3400-4400$ & Mont. 2219b & * \\
\hline $\begin{array}{l}\text { Dissanthelium macusaniense (E.H.L. Krause) R.C. Foster \& L.B. } \\
\text { Sm. }\end{array}$ & $\mathrm{h}$ & ms & $\mathrm{T}$ & $\mathrm{N}$ & $x$ & $3900-4400$ & Mont. 2420 & * \\
\hline Eragrostis glomerata (Walter) L.H. Dewey & $\mathrm{h}$ & $\mathrm{ms}$ & $\mathrm{Hc}$ & $\mathrm{N}$ & $\mathrm{x}$ & $3400-3500$ & Mont. 0783 & \\
\hline Eragrostis nigricans (Kunth) Steud. & $\mathrm{h}$ & $\mathrm{ms}$ & $\mathrm{Hc}$ & $\mathrm{N}$ & & $3400-4100$ & Mont. 2072 & * \\
\hline Festuca dolichophylla J. Presl & $\mathrm{h}$ & $\mathrm{ms}, \mathrm{pj}$ & Hc & $\mathrm{N}$ & & $3400-4600$ & Mont. 2028 & * \\
\hline Festuca orthophylla Pilg. & $\mathrm{h}$ & ms, pj, bf & $\mathrm{Hc}$ & $\mathrm{N}$ & & $3800-4700$ & Mont. 2470 & * \\
\hline Festuca sp. & $\mathrm{h}$ & $\mathrm{ms}$ & $\mathrm{Hc}$ & - & - & 3700 & Mont. 2334a & \\
\hline Hordeum muticum J. Presl. & $\mathrm{h}$ & $\mathrm{ms}$ & $\mathrm{T}$ & $\mathrm{N}$ & $x$ & 3500 & Mont. 0781 & \\
\hline Kikuyuochloa clandestina (Hochst. ex Chiov.) H. Scholz & $\mathrm{h}$ & $\mathrm{ms}$ & $\mathrm{Hc}$ & I & $x$ & $3400-3700$ & Mont. 1136 & \\
\hline Muhlenbergia peruviana (P. Beauv.) Steud. & $\mathrm{h}$ & ms, pj & $\mathrm{T}$ & $\mathrm{N}$ & & $3400-4400$ & Mont. 2109 & * \\
\hline Nassella asplundii Hitchc. & $\mathrm{h}$ & $\mathrm{ms}$ & $\mathrm{Hc}$ & $\mathrm{N}$ & $\mathrm{x}$ & 3500 & Mont. 0785 & \\
\hline Nassella inconspicua (J. Presl) Barkworth & $\mathrm{h}$ & ms, pj & $\mathrm{Hc}$ & $\mathrm{N}$ & $x$ & $3400-4500$ & Mont. 2333 & * \\
\hline Nassella pubiflora (Trin. \& Rupr.) E. Desv. & $\mathrm{h}$ & ms & $\mathrm{Hc}$ & $\mathrm{N}$ & & 3500 & Mont. 2011 & \\
\hline Poа anпuа L. & $\mathrm{h}$ & $\mathrm{ms}$ & $\mathrm{T}$ & $\mathrm{I}$ & $x$ & $3400-3700$ & $\mathrm{f} !$ & \\
\hline Poa candamoana Pilg. & $\mathrm{h}$ & ms, pj & $\mathrm{Hc}$ & $\mathrm{N}$ & $x$ & $3400-4500$ & Mont. 2055 & * \\
\hline Poa sp. & $\mathrm{h}$ & $\mathrm{ms}$ & $\mathrm{Hc}$ & - & - & $4400-4500$ & Mont. 2441 & \\
\hline Polypogon interruptus Kunth. & $\mathrm{h}$ & ms, pj, bf & $\mathrm{Hl}$ & $\mathrm{N}$ & & $3600-4100$ & Mont. 2335b & * \\
\hline Sporobolus indicus (L.) R. Br. & $\mathrm{h}$ & ms & $\mathrm{Hc}$ & $\mathrm{N}$ & $x$ & 3500 & Mont. 0782 & \\
\hline Stipa ichu (Ruiz \& Pav.) Kunth & $\mathrm{h}$ & $\mathrm{ms}, \mathrm{pj}, \mathrm{bf}$ & $\mathrm{Hc}$ & $\mathrm{N}$ & & $3400-4600$ & Mont. 2201 & * \\
\hline Stipa obtusa (Nees \& Meyen) Hitchc. & $\mathrm{h}$ & $\mathrm{ms}$ & $\mathrm{Hc}$ & $\mathrm{N}$ & $\mathrm{x}$ & $3700-3900$ & Mont. 2042 & * \\
\hline Stipa sp. & $\mathrm{h}$ & $\mathrm{ms}$ & $\mathrm{Hc}$ & - & - & $3400-3600$ & Mont. 2149 & * \\
\hline Tovarochloa peruviana T.D. Macfarl. \& But & $\mathrm{h}$ & pj & $\mathrm{T}$ & $\mathrm{E}$ & $\mathrm{x}$ & $4400-4500$ & Mont. 2428a & \\
\hline \multicolumn{9}{|l|}{ Zannichelliaceae } \\
\hline Zannichellia andina Holm-Niels. \& R.R. Haynes & $\mathrm{h}$ & bf & $\mathrm{Hd}$ & $\mathrm{N}$ & & $4200-4400$ & Mont. 2600 & \\
\hline
\end{tabular}

Amaranthaceae

Alternanthera caracasana Kunth.

Atriplex herzogii Standl.

Chenopodium ambrosiodes L.

Chenopodium incisum Poir.

Chenopodium petiolare Kunth

Gomphrena meyeniana Walp.

Guilleminea densa (Humb. \& Bonpl. ex Schult.) Moq.

Sarcocornia pulvinata (R.E. Fr.) A. J. Scott

Anacardiaceae

Schinus molle L.

Apiaceae

Ammi visnaga (L.) Lam

Azorella compacta Phil.

Azorella diapensioides A. Gray

Azorella sp.

Bowlesia lobata Ruiz \& Pav.

Bowlesia sodiroana $\mathrm{H}$. Wolff

Cyclospermum leptophyllum (Pers.) Sprague ex Britton \& P.

Wilson

Conium maculatum L.

Daucus montanus Humb. \& Bonpl. ex Spreng.

Lilaeopsis macloviana (Gand.) A.W. Hill

Oreomyrrhis andicola (Kunth) Endl. ex Hook. f.

\section{Asclepiadaceae}

Philibertia lysimachioides (Wedd.) T. Mey

\section{Asteraceae}

Sarcostemma solanoides (Kunth) Decne.

Acanthoxanthium spinosum (L.) Fourr.

Achyrocline alata (Kunth) DC.

Achyrocline ramosissima Britton ex Rusby

Ageratina azangaroensis (Schultz-Bip) King H. Robinson

Ageratina sternbergiana (DC.) R.M. King \& H. Rob.

Ambrosia arborescens Mill.

Aristeguietia ballii (Oliv.) R.M. King \& H. Rob.

Baccharis alpina Kunth

Baccharis caespitosa (Ruiz \& Pav.) Pers.

Baccharis genistelloides (Lam.) Pers.

Baccharis petiolata DC.

\begin{tabular}{|c|c|c|c|c|c|}
\hline $\mathrm{ms}$ & $\mathrm{Hc}$ & $\mathrm{N}$ & $x$ & $3400-3800$ & Mont. 2604 \\
\hline $\mathrm{ms}$ & $\mathrm{Hc}$ & $\mathrm{N}$ & & $3400-3400$ & Mont. 2211 \\
\hline $\mathrm{ms}$ & $\mathrm{Hc}$ & I & & $3400-3800$ & Mont. 0583 \\
\hline $\mathrm{ms}$ & $\mathrm{T}$ & $\mathrm{N}$ & $x$ & $3500-3600$ & Mont. 2240 \\
\hline $\mathrm{ms}$ & $\mathrm{T}$ & I & & $3400-3600$ & Mont. 0602 \\
\hline ms, pj & $\mathrm{Hc}$ & $\mathrm{N}$ & $x$ & $3600-4000$ & Mont. 2031 \\
\hline $\mathrm{ms}$ & Hc & $\mathrm{N}$ & $x$ & $3500-3600$ & Mont. 2020 \\
\hline $\mathrm{pj}$ & $\mathrm{Hl}$ & $\mathrm{N}$ & $x$ & $4000-4200$ & $\mathrm{f} !$ \\
\hline $\mathrm{ms}$ & $\mathrm{F}$ & $\mathrm{N}$ & & $3400-3400$ & Mont. 1209 \\
\hline $\mathrm{ms}$ & $\mathrm{T}$ & I & & $3500-3650$ & Mont. 0980 \\
\hline pj & $\mathrm{Cm}$ & $\mathrm{N}$ & & $4400-4700$ & Mont. 2410 \\
\hline pj, bf & $\mathrm{Cm}$ & $\mathrm{N}$ & $x$ & $4200-4700$ & Mont. 2429 \\
\hline $\mathrm{pj}$ & $\mathrm{T}$ & - & - & 3850 & Mont. 2132 \\
\hline $\mathrm{ms}$ & $\mathrm{T}$ & $\mathrm{N}$ & $x$ & $3800-4000$ & Mont. 2542 \\
\hline $\mathrm{ms}$ & $\mathrm{T}$ & $\mathrm{N}$ & & $3500-3900$ & Mont. 2092 \\
\hline $\mathrm{ms}$ & $\mathrm{T}$ & $\mathrm{N}$ & & $3400-3900$ & Mont. 0547 \\
\hline $\mathrm{ms}$ & $\mathrm{T}$ & I & $x$ & $3400-3500$ & Mont. 0965 \\
\hline $\mathrm{ms}$ & $\mathrm{T}$ & $\mathrm{N}$ & $x$ & $3500-3700$ & Mont. 0987 \\
\hline $\mathrm{ms}, \mathrm{bf}$ & $\mathrm{Hd}$ & $\mathrm{N}$ & $x$ & $3600-4000$ & Mont. 2539 \\
\hline $\mathrm{pj}$ & Hc & $\mathrm{N}$ & $\mathrm{x}$ & $4300-4700$ & Mont. 2397 \\
\hline ms, pj & $\mathrm{Cm}$ & $\mathrm{N}$ & $x$ & $3700-4050$ & Mont. 2331 \\
\hline $\mathrm{ms}$ & $\mathrm{Hc}$ & $\mathrm{N}$ & & $3400-3700$ & Mont. 2086 \\
\hline $\mathrm{ms}$ & $\mathrm{Hc}$ & $\mathrm{N}$ & & $3400-3700$ & Mont. 0589 \\
\hline $\mathrm{ms}$ & $\mathrm{Hc}$ & $\mathrm{N}$ & & $3500-4200$ & Mont. 1254 \\
\hline $\mathrm{ms}, \mathrm{pj}$ & Hc & $\mathrm{N}$ & $x$ & $3400-3700$ & Mont. 2609 \\
\hline $\mathrm{ms}, \mathrm{pj}$ & Hc & $\mathrm{N}$ & $x$ & $3700-4000$ & Mont. 2032 \\
\hline ms, pj & $\mathrm{Nf}$ & $\mathrm{N}$ & $x$ & $3700-4200$ & Mont. 2610 \\
\hline $\mathrm{ms}$ & $\mathrm{Nf}$ & $\mathrm{N}$ & & 3500 & Mont. 0571 \\
\hline $\mathrm{ms}$ & $\mathrm{Nf}$ & $\mathrm{E}$ & $x$ & $3500-4000$ & Mont. 2247 \\
\hline pj & $\mathrm{Cm}$ & $\mathrm{N}$ & $x$ & 4100 & Mont. 2608 \\
\hline pj & $\mathrm{Cm}$ & $\mathrm{N}$ & $x$ & $3900-4200$ & Mont. 2182 \\
\hline pj & $\mathrm{Hc}$ & $\mathrm{N}$ & $x$ & $3900-4200$ & Mont. 2554 \\
\hline $\mathrm{ms}$ & $\mathrm{Nf}$ & $\mathrm{N}$ & & $3400-3700$ & Mont. 0506 \\
\hline
\end{tabular}

(Continúa...) 


\begin{tabular}{|c|c|c|c|c|c|c|c|c|}
\hline Taxón & FC & FE & FB & St & NR & Altitud (m) & Voucher & \\
\hline Baccharis tricuneata (L. f) Pers. & $\mathrm{s}$ & $\mathrm{ms}, \mathrm{pj}$ & $\mathrm{Nf}$ & $\mathrm{N}$ & & $3700-4700$ & Mont. 2413 & * \\
\hline Belloa longifolia (Cuatrec. \& Arist.) Sagást. \& Dillon & $\mathrm{h}$ & $\mathrm{ms}$ & $\mathrm{Hc}$ & $\mathrm{N}$ & $\mathrm{x}$ & $3400-4100$ & Mont. 2547 & * \\
\hline Belloa piptolepis (Wedd.) Cabrera & $\mathrm{h}$ & $\mathrm{ms}, \mathrm{pj}$ & $\mathrm{Cm}$ & $\mathrm{N}$ & & $3400-4600$ & Mont. 2184 & * \\
\hline Belloa schultzii (Wedd.) Cabrera & $\mathrm{h}$ & $\mathrm{ms}, \mathrm{pj}$ & $\mathrm{Cm}$ & $\mathrm{N}$ & & $3900-4500$ & Mont. 2046 & * \\
\hline Bidens andicola Kunth & $\mathrm{h}$ & $\mathrm{ms}$ & $\mathrm{Hc}$ & $\mathrm{N}$ & $\mathrm{x}$ & $3400-3900$ & Mont. 0530 & * \\
\hline Bidens pilosa $\mathrm{L}$. & $\mathrm{h}$ & $\mathrm{ms}$ & $\mathrm{T}$ & I & & 3500 & Mont. 0963 & \\
\hline Chaetanthera sp. & $\mathrm{h}$ & pj & $\mathrm{Hc}$ & - & & 4500 & Mont. 2472 & \\
\hline Chaptalia cf. similis R.E. Fr. & $\mathrm{h}$ & ms & $\mathrm{Hc}$ & $\mathrm{N}$ & $\mathrm{x}$ & $3600-3800$ & Mont. 2148 & \\
\hline Chersodoma jodopappa (Sch. Bip.) Cabrera & $\mathrm{s}$ & $\mathrm{ms}, \mathrm{pj}$ & $\mathrm{Nf}$ & $\mathrm{N}$ & & $3700-4400$ & Mont. 1003 & * \\
\hline Chuquiraga rotundifolia Wedd. & $\mathrm{s}$ & $\mathrm{ms}$ & $\mathrm{Nf}$ & $\mathrm{N}$ & & $3500-3700$ & Mont. 2231 & * \\
\hline Conyza artemisiifolia Meyen \& Walp. & $\mathrm{h}$ & $\mathrm{ms}, \mathrm{pj}$ & $\mathrm{T}$ & $\mathrm{N}$ & & $3700-4300$ & Mont. 2274 & * \\
\hline $\begin{array}{l}\text { Conyza sumatrensis var. leiotheca (S.F. Blake) Pruski \& G. } \\
\text { Sancho }\end{array}$ & $\mathrm{h}$ & $\mathrm{ms}$ & $\mathrm{Hc}$ & $\mathrm{N}$ & & $3400-3700$ & Mont. 2101 & * \\
\hline $\begin{array}{l}\text { Sancho } \\
\text { Cotula mexicana (DC.) Cabrera }\end{array}$ & $\mathrm{h}$ & $\mathrm{ms}$ & $\mathrm{Hd}$ & I & & $3400-4400$ & Mont. 2016a & * \\
\hline Diplostephium meyenii (Sch. Bip. ex Wedd.) S.F. Blake & $\mathrm{s}$ & $\mathrm{ms}$ & $\mathrm{Nf}$ & $\mathrm{N}$ & & $3400-3600$ & Mont. 2213 & * \\
\hline Erigeron pazensis Sch. Bip. ex Rusby & $\mathrm{h}$ & $\mathrm{ms}$ & $\mathrm{Hc}$ & $\mathrm{N}$ & $\mathrm{x}$ & $3400-4200$ & Mont. 2150 & * \\
\hline Facelis plumosa (Wedd.) Sch. Bip. & $\mathrm{h}$ & ms, pj & $\mathrm{T}$ & $\mathrm{N}$ & $\mathrm{x}$ & $3400-3900$ & Mont. 2621 & * \\
\hline Galinsoga mandonii Sch. Bip & $\mathrm{h}$ & $\mathrm{ms}$ & $\mathrm{T}$ & $\mathrm{N}$ & $\mathrm{x}$ & $3400-3600$ & Mont. 2127b & * \\
\hline Galinsoga sp. & $\mathrm{h}$ & $\mathrm{pj}$ & $\mathrm{T}$ & - & & 3800 & Mont. 2500 & \\
\hline Gamochaeta americana (Mill.) Wedd. & $\mathrm{h}$ & ms & $\mathrm{Hc}$ & $\mathrm{N}$ & & $3450-3650$ & Mont. 2188 & * \\
\hline Gamochaeta cf. humilis Wedd. & $\mathrm{h}$ & $\mathrm{ms}$ & $\mathrm{Hc}$ & $\mathrm{N}$ & & $3450-3500$ & Mont. 2064 & * \\
\hline Gamochaeta purpurea (L.) Cabrera & $\mathrm{h}$ & $\mathrm{ms}, \mathrm{pj}$ & $\mathrm{Hc}$ & $\mathrm{N}$ & & $3600-3900$ & Mont. 0796 & * \\
\hline Gnaphalium dombeyanum DC. & hs & $\mathrm{ms}, \mathrm{pj}$ & $\mathrm{Hc}$ & $\mathrm{N}$ & & $3500-4100$ & Mont. 2140 & * \\
\hline Gnaphalium lacteum Meven \& Walp. & $\mathrm{h}$ & $\mathrm{ms}$ & $\mathrm{Hc}$ & $\mathrm{N}$ & $\mathrm{x}$ & 3400 & Mont. 2206b & \\
\hline Gnaphalium polium Wedd. & $\mathrm{h}$ & $\mathrm{ms}$ & $\mathrm{Hc}$ & $\mathrm{N}$ & $\mathrm{x}$ & 3600 & Mont. 2098 & \\
\hline Gochnatia arequipensis Sandwith & $\mathrm{s}$ & $\mathrm{ms}$ & $\mathrm{Nf}$ & $\mathrm{E}$ & $x$ & $3400-3600$ & Mont. 2346 & * \\
\hline Helogyne ferreyrae R.M. King \& H. Rob. & $\mathrm{s}$ & $\mathrm{ms}$ & $\mathrm{Nf}$ & $\mathrm{E}$ & $\mathrm{x}$ & $3500-3650$ & Mont. 0739 & * \\
\hline Heterosperma diversifolium Kunth & $\mathrm{h}$ & $\mathrm{ms}$ & $\mathrm{T}$ & $\mathrm{N}$ & $\mathrm{x}$ & $3400-3800$ & Mont. 0758 & * \\
\hline Hieracium peruanum Fr. & $\mathrm{h}$ & $\mathrm{ms}$ & $\mathrm{Hc}$ & $\mathrm{E}$ & $\mathrm{x}$ & $3700-4000$ & Mont. 0684 & \\
\hline Hieracium cf. streptochaetum Zahn & $\mathrm{h}$ & $\mathrm{ms}, \mathrm{pj}$ & $\mathrm{Hc}$ & $\mathrm{N}$ & $\mathrm{x}$ & $3700-4200$ & Mont. 2029 & * \\
\hline Hypochaeris chillensis (Kunth) Britton & $\mathrm{h}$ & $\mathrm{ms}, \mathrm{pj}$ & $\mathrm{T}$ & $\mathrm{N}$ & $\mathrm{x}$ & $3400-4200$ & Mont. 2121 & * \\
\hline Hypochaeris echegarayi Hieron. & $\mathrm{h}$ & $\mathrm{ms}, \mathrm{pj}$ & $\mathrm{Hc}$ & $\mathrm{N}$ & $\mathrm{x}$ & $3600-3750$ & $\mathrm{f} !$ & \\
\hline Hypochaeris meyeniana (Walp.) Benth. \& Hook. f. ex Griseb. & $\mathrm{h}$ & $\mathrm{ms}$ & $\mathrm{Hc}$ & $\mathrm{N}$ & $\mathrm{x}$ & 3750 & $\mathrm{f} !$ & \\
\hline Hypochaeris taraxacoides (Meyen \& Walp.) Ball & $\mathrm{h}$ & $\mathrm{ms}, \mathrm{pj}, \mathrm{bf}$ & $\mathrm{Hc}$ & $\mathrm{N}$ & $\mathrm{x}$ & $3400-4700$ & Mont. 0597 & * \\
\hline Hypochaeris sp. 1 & $\mathrm{~h}$ & pj & $\mathrm{Hc}$ & - & & $4000-4400$ & Mont. 2459 & * \\
\hline Hypochaeris sp. 2 & $\mathrm{~h}$ & pj & $\mathrm{Hc}$ & - & & 4300 & Mont. 2593 & \\
\hline Hypochaeris sp. 3 & $\mathrm{~h}$ & pj & $\mathrm{Hc}$ & - & & $3700-4000$ & Mont. 2041 & \\
\hline Lophopappus foliosus Rusby & $\mathrm{s}$ & ms & $\mathrm{Nf}$ & $\mathrm{N}$ & $\mathrm{x}$ & $3400-3600$ & Mont. 2214 & * \\
\hline Loricaria graveolens (Sch. Bip.) Wedd. & $\mathrm{s}$ & pj & $\mathrm{Nf}$ & $\mathrm{N}$ & $\mathrm{x}$ & $4050-4200$ & Mont. 2056 & * \\
\hline Lucilia cf. conoidea Wedd. & $\mathrm{h}$ & pj & $\mathrm{Cm}$ & $\mathrm{N}$ & $\mathrm{x}$ & $4050-4250$ & Mont. 2051 & * \\
\hline Mniodes aretioides (Wedd.) Cuatrec. & $\mathrm{s}$ & $\mathrm{pj}$ & $\mathrm{Cm}$ & $\mathrm{E}$ & $\mathrm{x}$ & $4450-4700$ & Mont. 2181 & * \\
\hline Mutisia acuminata var. hirsuta (Meyen) Cabrera & $\mathrm{s}$ & $\mathrm{ms}$ & $\mathrm{Nf}$ & $\mathrm{N}$ & & $3400-3600$ & Mont. 0500 & \\
\hline Mutisia lanigera Wedd. & $\mathrm{h}$ & $\mathrm{ms}$ & $\mathrm{Hc}$ & $\mathrm{N}$ & $\mathrm{x}$ & 4050 & Mont. 1173 & \\
\hline Mutisia orbignyana Wedd. & $\mathrm{s}$ & $\mathrm{ms}, \mathrm{pj}$ & $\mathrm{Nf}$ & $\mathrm{N}$ & $\mathrm{x}$ & $3600-4200$ & Mont. 2355 & * \\
\hline Ophryosporus heptanthus (Sch. Bip. ex Wedd.) R.M. King \& H. Rob. & $\mathrm{s}$ & $\mathrm{ms}$ & $\mathrm{Nf}$ & $\mathrm{N}$ & & $3400-3800$ & Mont. 0704 & \\
\hline Oritrophium limnophilum (Sch. Bip.) Cuatrec. & $\mathrm{h}$ & pj & $\mathrm{T}$ & $\mathrm{N}$ & $\mathrm{x}$ & 4050 & Mont. 2194b & \\
\hline Parastrephia lucida (Meyen) Cabrera & $\mathrm{s}$ & $\mathrm{pj}$, bf & $\mathrm{Nf}$ & $\mathrm{N}$ & $\mathrm{x}$ & $4400-4700$ & Mont. 2364 & * \\
\hline Parastrephia quadrangularis (Meyen) Cabrera & $\mathrm{s}$ & $\mathrm{ms}, \mathrm{pj}, \mathrm{bf}$ & $\mathrm{Nf}$ & $\mathrm{N}$ & & $3400-4400$ & Mont. 2329 & * \\
\hline Perezia coerulescens Wedd. & $\mathrm{h}$ & pj & $\mathrm{Hc}$ & $\mathrm{N}$ & $x$ & $3900-4500$ & Mont. 2279 & * \\
\hline Perezia multiflora (Bonpl.) Less. & $\mathrm{h}$ & $\mathrm{ms}, \mathrm{pj}$ & $\mathrm{T}$ & $\mathrm{N}$ & $x$ & $4000-4500$ & Mont. 0923 & * \\
\hline Perezia cf. pungens (Bonpl.) Less. & $\mathrm{h}$ & $\mathrm{ms}$ & $\mathrm{Hc}$ & $\mathrm{N}$ & $\mathrm{x}$ & $3550-3900$ & Mont. 2226 & * \\
\hline Perezia sublyrata Domke & $\mathrm{h}$ & $\mathrm{ms}$ & $\mathrm{Hc}$ & $\mathrm{N}$ & $x$ & $3700-3850$ & Mont. 2565 & \\
\hline Perezia sp. & $\mathrm{h}$ & bf & $\mathrm{Hl}$ & - & & 3800 & Mont. 2495 & \\
\hline Plazia daphnoides Wedd. & $\mathrm{s}$ & $\mathrm{ms}$ & $\mathrm{Nf}$ & $\mathrm{N}$ & $x$ & $3500-4000$ & Mont. 2001 & * \\
\hline Polyachyrus sphaerocephalus D. Don & hs & $\mathrm{ms}$ & $\mathrm{Nf}$ & $\mathrm{N}$ & & $3400-3600$ & Mont. 0975 & \\
\hline Proustia berberidifolia (Cuatrec.) Ferreyra & $\mathrm{s}$ & $\mathrm{ms}, \mathrm{pj}$ & $\mathrm{Nf}$ & $\mathrm{E}$ & & $3450-3900$ & Mont. 1199 & * \\
\hline Proustia cuneifolia D. Don & $\mathrm{s}$ & $\mathrm{ms}$ & $\mathrm{Nf}$ & $\mathrm{N}$ & $x$ & $3400-4000$ & Mont. 0501 & * \\
\hline Senecio adenophyllus Meyen \& Walp. & hs & $\mathrm{ms}$ & $\mathrm{Hc}$ & $\mathrm{N}$ & $\mathrm{x}$ & $3600-4100$ & Mont. 0693 & * \\
\hline Senecio arnaldii Cabrera & hs & $\mathrm{ms}$ & $\mathrm{Nf}$ & $\mathrm{E}$ & $x$ & $3400-3750$ & Mont. 0791 & * \\
\hline Senecio bolivarianus Cuatrec. & $\mathrm{h}$ & pj & $\mathrm{Hc}$ & $\mathrm{N}$ & $\mathrm{x}$ & $4000-4600$ & Mont. 2307 & * \\
\hline Senecio breviscapus DC. & $\mathrm{h}$ & pj & $\mathrm{Hl}$ & $\mathrm{N}$ & $\mathrm{x}$ & $3900-4000$ & Mont. 2285 & \\
\hline Senecio candollei Wedd. & $\mathrm{h}$ & pj & $\mathrm{Hc}$ & $\mathrm{N}$ & $x$ & $4000-4700$ & Mont. 2314 & * \\
\hline Senecio cf. chachaniensis Cuatrec. & $\mathrm{s}$ & pj & $\mathrm{Nf}$ & $\mathrm{E}$ & $\mathrm{x}$ & $4450-4700$ & Mont. 2406 & \\
\hline Senecio evacoides Sch. Bip. & hs & pj & $\mathrm{Nf}$ & $\mathrm{N}$ & $\mathrm{x}$ & $4500-4700$ & Mont. 2404 & \\
\hline Senecio cf. ferreyrae Cabrera & $\mathrm{s}$ & $\mathrm{ms}, \mathrm{pj}$ & $\mathrm{Nf}$ & $\mathrm{E}$ & $x$ & $3800-3950$ & Mont. 2146 & \\
\hline Senecio gamolepis Cabrera & $\mathrm{h}$ & $\mathrm{pj}$ & $\mathrm{Cm}$ & $\mathrm{E}$ & $\mathrm{x}$ & $4300-4500$ & Mont. 2429 & * \\
\hline Senecio herrerae Cabrera & hs & ms & $\mathrm{Hc}$ & $\mathrm{N}$ & $\mathrm{x}$ & 3500 & Mont. 2099 & \\
\hline Senecio nutans Sch. Bip. & $\mathrm{s}$ & pj & $\mathrm{Nf}$ & $\mathrm{N}$ & $\mathrm{x}$ & $4000-4700$ & Mont. 2263 & * \\
\hline Senecio phylloleptus Cuatrec. & hs & $\mathrm{ms}$ & $\mathrm{Hc}$ & $\mathrm{N}$ & & 3500 & Mont. 1222 & \\
\hline
\end{tabular}

(Continúa...) 
Apéndice 1. Continuación

\section{Taxón}

Senecio rhizomatus Rusby

Senecio rudbeckiifolius Meyen \& Walp.

Senecio rufescens DC.

Senecio serratifolius (Meyen \& Walp.) Cuatrec.

Senecio spinosus DC.

Senecio cf. sublutescens Cuatrec.

Senecio tovari Cabrera

Senecio vulgaris $\mathrm{L}$.

Senecio sp. 1

Senecio sp. 2

Sonchus oleraceus L.

Stevia macbridei B.L. Rob.

Stevia mandonii Sch. Bip.

Tagetes filiifolia Lag.

Tagetes minuta $\mathrm{L}$

Tagetes multiflora Kunth

Tanacetum vulgare fo. crispum (L.) Fernald

Taraxacum officinale F.H. Wigg.

Vasquezia oppositifolia (Lag.) S.F. Blake

Viguiera lanceolata Britton

Werneria cf. apiculata Sch. Bip.

Werneria glaberrima Phil.

Werneria nubigena Kunth

Werneria cf. pectinata Lingelsh.

Werneria pygmaea Gillies ex Hook. \& Arn.

Werneria sp.

Xenophyllum poposum (Phil.) V.A. Funk

\section{Basellaceae}

Anredera diffusa (Moq.) Sperling

Ullucus tuberosus subsp. aborigineus (Bruecher) Sperling

\section{Boraginaceae}

Cryptantha peruviana I.M. Johnst.

Heliotropium microstachyum Ruiz \& Pav.

Pectocarya cf. anomala I.M. Johnst.

Plagiobothrys humilis (Ruiz \& Pav.) I.M. Johnst.

Plagiobothrys kunthii (Walp.) I.M. Johnst.

\section{Brassicaceae}

Brassica rapa $\mathrm{L}$.

Brayopsis calycina (Desv.) Gilg \& Muschl.

Capsella bursa-pastoris (L.) Medik.

Descurainia cf. athroocarpa (A. Gray) O. E. Schulz

Descurainia cf. depressa (Phil.) Prantl

Descurainia myriophylla (Willd. ex DC.) R.E. Fr.

Descurainia sp.

Draba macleanii Hook. f.

Exhalimolobos pazensis (Rusby) Al-Shehbaz \& C.D. Bailey

Exhalimolobos weddelii (E. Fourn.) Al-Shehbaz \& C.D. Bailey

Lepidium raimondii O.E. Shultz

Lepidium weddellii O.E. Schulz

Lepidium sp. 1

Lepidium sp. 2

Mancoa hispida Wedd.

Matthiola incana (L.) R. Br.

Mostacillastrum gracile (Wedd.) Al-Shehbaz

Sisymbrium officinale (L.) Scop.

Sisymbrium peruvianum DC.

Thlaspi arvense $\mathrm{L}$.

Weberbauera peruviana (DC.) Al-Shehbaz

Weberbauera spathulifolia (A. Gray) O.E. Schulz

\section{Cactaceae}

Austrocilyndropuntia subulata (Muehlenpf.) Backeb.

Borzicactus hendriksenianus (Backeb.) Kimnach

Cumulopuntia ignota (Backeb.) F. Ritter

Cumulopuntia mistiensis (Backeb.) E.F. Anderson

Cumulopuntia zehnderi (Rauh \& Backeb.) F. Ritter.

Cumulopuntia sp.

Cylindropuntia rosea (DC.) Backeb.

Echinopsis pampana (Britton \& Rose) D.R. Hunt

$\begin{array}{lllllll}\text { FC } & \text { FE } & \text { FB } & \text { St } & \text { NR } & \text { Altitud (m) } & \text { Voucher }\end{array}$

\begin{tabular}{|c|c|c|c|c|c|c|c|}
\hline h & $\mathrm{ms}$ & $\mathrm{Hl}$ & $\mathrm{N}$ & $\mathrm{x}$ & 3550 & Mont. 1160 & \\
\hline s & $\mathrm{ms}$ & $\mathrm{Nf}$ & $\mathrm{N}$ & & $3450-3600$ & Mont. 0507 & * \\
\hline hs & $\mathrm{pj}, \mathrm{bf}$ & $\mathrm{Hc}$ & $\mathrm{N}$ & $x$ & $4450-4650$ & Mont. 2395 & \\
\hline $\mathrm{h}$ & bf & $\mathrm{Hl}$ & $\mathrm{N}$ & $x$ & 4450 & Mont. 2466 & \\
\hline $\mathrm{s}$ & pj & $\mathrm{Nf}$ & $\mathrm{N}$ & $x$ & $4200-4600$ & Mont. 2462 & \\
\hline s & $\mathrm{ms}, \mathrm{pj}$ & $\mathrm{Nf}$ & $\mathrm{N}$ & $x$ & $3750-4000$ & Mont. 2035 & * \\
\hline s & $\mathrm{ms}$ & $\mathrm{Nf}$ & $\mathrm{E}$ & $x$ & $3400-3600$ & Mont. 2212 & * \\
\hline $\mathrm{h}$ & $\mathrm{ms}$ & $\mathrm{T}$ & $\mathrm{Co}$ & $x$ & $3400-3500$ & Mont. 0512 & \\
\hline h & pj & $\mathrm{Nf}$ & - & & 3500 & Mont. 2246 & \\
\hline h & $\mathrm{ms}$ & $\mathrm{Cm}$ & - & & $4400-4500$ & Mont. 2400 & \\
\hline h & $\mathrm{ms}$ & $\mathrm{T}$ & Co & & $3400-3600$ & Mont. 0644 & * \\
\hline $\mathrm{s}$ & $\mathrm{ms}$ & $\mathrm{Hc}$ & E & $x$ & $3400-4100$ & Mont. 2233 & * \\
\hline s & $\mathrm{ms}$ & $\mathrm{Hc}$ & $\mathrm{N}$ & $x$ & $3800-4100$ & Mont. 2561 & * \\
\hline h & $\mathrm{ms}$ & $\mathrm{T}$ & $\mathrm{N}$ & $\mathrm{x}$ & $3600-3900$ & Mont. 1010 & \\
\hline h & $\mathrm{ms}$ & $\mathrm{T}$ & $\mathrm{N}$ & $x$ & $3400-3600$ & Mont. 0973 & \\
\hline h & ms, pj & $\mathrm{T}$ & $\mathrm{N}$ & & $3400-4200$ & Mont. 0974 & \\
\hline h & $\mathrm{ms}, \mathrm{pj}$ & $\mathrm{Hl}$ & I & $x$ & $3700-3900$ & Mont. 0511 & \\
\hline h & $\mathrm{ms}$ & $\mathrm{T}$ & Co & & $3400-3600$ & Mont. 1229 & \\
\hline h & $\mathrm{ms}$ & $\mathrm{Hc}$ & $\mathrm{N}$ & & $3500-3900$ & Mont. 2230 & * \\
\hline $\mathrm{s}$ & $\mathrm{ms}$ & $\mathrm{Nf}$ & $\mathrm{N}$ & $\mathrm{x}$ & $3400-3700$ & Mont. 0631 & \\
\hline h & $\mathrm{pj}, \mathrm{bf}$ & $\mathrm{T}$ & $\mathrm{N}$ & $\mathrm{x}$ & $4000-4500$ & Mont. 2289 & * \\
\hline h & $\mathrm{pj}, \mathrm{bf}$ & $\mathrm{Hc}$ & $\mathrm{N}$ & $x$ & 4000 & Mont. 2311 & \\
\hline h & pj & $\mathrm{Hc}$ & $\mathrm{N}$ & $\mathrm{x}$ & $4350-4400$ & Mont. 2383 & \\
\hline h & $\mathrm{pj}, \mathrm{bf}$ & $\mathrm{Cm}$ & $\mathrm{N}$ & $x$ & $4100-4700$ & Mont. 2254 & * \\
\hline h & $\mathrm{pj}, \mathrm{bf}$ & $\mathrm{Hl}$ & $\mathrm{N}$ & $x$ & $4200-4500$ & Mont. 2452 & * \\
\hline h & pj & $\mathrm{Hc}$ & - & & $4000-4200$ & Mont. 2319 & \\
\hline $\mathrm{s}$ & $\mathrm{pj}$ & $\mathrm{Cm}$ & $\mathrm{N}$ & $\mathrm{x}$ & $4450-4600$ & Mont. 2484 & * \\
\hline h & $\mathrm{ms}$ & $\mathrm{Hc}$ & $\mathrm{N}$ & & $3400-3750$ & Mont. 2344 & * \\
\hline h & $\mathrm{ms}$ & G & $\mathrm{N}$ & $\mathrm{x}$ & $3500-3600$ & Mont. 2115 & \\
\hline h & $\mathrm{ms}$ & $\mathrm{T}$ & $\mathrm{N}$ & & $3450-3600$ & Mont. 2104 & * \\
\hline $\mathrm{h}$ & $\mathrm{ms}$ & $\mathrm{Hc}$ & $\mathrm{N}$ & & $3450-3700$ & Mont. 2115 & * \\
\hline h & $\mathrm{ms}$ & $\mathrm{T}$ & $\mathrm{E}$ & $\mathrm{x}$ & 4050 & Mont. 2194b & \\
\hline h & $\mathrm{ms}, \mathrm{pj}$ & $\mathrm{T}$ & $\mathrm{N}$ & $\mathrm{x}$ & $4000-4400$ & Mont. 2379 & * \\
\hline
\end{tabular}

\section{0-3600 Mont. 0797}

\section{0-4600 Mont. 2178a}

3500-3700 Mont. 2487

3550-3800 Mont. 1187

3800-4500 Mont. 2295

3400-3800 Mont. 0724

$4500 \quad$ Mont. 0940

4450-4550 Mont. 2439

3600-3700 Mont. 0797

3400-3550 Mont. 2124

3550-3600 Mont. 2008

3400-3600 Mont. 1234

3550-3600 Mont. 2006

4400-4500 Mont. 2367

3650-4700 Mont. 2160b

3450-3600 Mont. 1155

3450-3600 Mont. 2217

3400-3500 Mont. 0690

3500-3600 Mont. 0741

3750-3800 Mont. 2569

$3850-4150$

Mont. 2252 *
Mont. 2414 *

$4300-4700$

$\begin{array}{ll}\mathrm{su} & \mathrm{m} \\ \mathrm{su} & \mathrm{m} \\ \mathrm{su} & \mathrm{m} \\ \mathrm{su} & \mathrm{m} \\ \mathrm{su} & \mathrm{m} \\ \mathrm{su} & \mathrm{m} \\ \mathrm{su} & \mathrm{m} \\ \mathrm{su} & \mathrm{ms}\end{array}$

$\mathrm{ms}$
$\mathrm{ms}$
$\mathrm{ms}$
$\mathrm{ms}$
$\mathrm{ms}$
$\mathrm{ms}$
$\mathrm{ms}$
$\mathrm{ms}, \mathrm{pj}$

3400-3700

$3450-3700$

3400-3700

3550-3650

3950-4050

3580

3400-3700

$3400-4200$ 


\begin{tabular}{|c|c|c|c|c|c|c|c|c|}
\hline Taxón & FC & FE & FB & St & NR & Altitud (m) & Voucher & \\
\hline Echinopsis schoenii (Rauh \& Backeb.) Friedrich \& G.D. Rowley & su & $\mathrm{ms}$ & $\mathrm{S}$ & $\mathrm{E}$ & $\mathrm{x}$ & $3400-3600$ & $\mathrm{f} !$ & \\
\hline $\begin{array}{l}\text { Echinopsis tulhuayacensis (Ochoa ex Backeb.) Friedrich \& G. D. } \\
\text { Rowley }\end{array}$ & su & ms & S & $\mathrm{E}$ & $\mathrm{x}$ & $3450-3600$ & $\mathrm{f} !$ & \\
\hline Echinopsis sp. & su & pj & S & - & - & $4000-4100$ & $\mathrm{f} !$ & \\
\hline Neowerdermannia chilensis subsp. peruviana (F. Ritter) Ostolaza & su & $\mathrm{ms}, \mathrm{pj}$ & S & $\mathrm{E}$ & & $3500-4100$ & $\mathrm{f} !$ & \\
\hline Opuntia ignescens Vaupel & su & $\mathrm{ms}, \mathrm{pj}$ & S & $\mathrm{N}$ & & $3700-4500$ & $\mathrm{f} !$ & \\
\hline Opuntia pubescens J.C. Wendl. ex Pfeiff. & su & $\mathrm{ms}$ & $\mathrm{S}$ & $\mathrm{N}$ & $\mathrm{x}$ & 3500 & $\mathrm{f} !$ & \\
\hline Tunilla soehrensii (Britton \& Rose) D.R. Hunt \& Iliff. & su & $\mathrm{ms}$ & $\mathrm{s}$ & $\mathrm{N}$ & & $3400-3750$ & $\mathrm{f} !$ & \\
\hline \multicolumn{9}{|l|}{ Calceolariaceae } \\
\hline Calceolaria inamoena Kraenzl. & $\mathrm{s}$ & $\mathrm{ms}, \mathrm{pj}$ & $\mathrm{Nf}$ & $\mathrm{N}$ & & $3400-4100$ & Mont. 2204 & * \\
\hline Calceolaria lobata Cav. & hs & $\mathrm{ms}, \mathrm{pj}$ & $\mathrm{Hc}$ & $\mathrm{N}$ & & $3500-4000$ & Mont. 2059 & \\
\hline Calceolaria pisacomensis Meyen ex Walp. & $\mathrm{s}$ & $\mathrm{ms}$ & $\mathrm{Nf}$ & $\mathrm{E}$ & & 3450 & Mont. 1223 & * \\
\hline Calceolaria plectranthifolia Walp. & $\mathrm{h}$ & ms, pj & $\mathrm{Hl}$ & $\mathrm{N}$ & & $3500-3900$ & Mont. 2013 & * \\
\hline \multicolumn{9}{|l|}{$\begin{array}{l}\text { Callitrichaceae } \\
\text { P. }\end{array}$} \\
\hline \multicolumn{9}{|l|}{ Campanulaceae } \\
\hline Lobelia oligophylla (Wedd.) Lammers & $\mathrm{h}$ & bf & $\mathrm{Hl}$ & $\mathrm{N}$ & $\mathrm{x}$ & $4400-4500$ & Mont. 2450 & \\
\hline $\begin{array}{l}\text { Lysipomia cf. glandulifera (Schlechtendal ex Weddell) Schltdl. ex } \\
\text { E. Wimm. }\end{array}$ & $\mathrm{h}$ & bf & $\mathrm{Hl}$ & - & $x$ & 4050 & Mont. 0697 & \\
\hline Lysipomia sp. & s & $\mathrm{pj}$ & $\mathrm{Hl}$ & - & - & 4400 & Mont. 2456 & \\
\hline Wahlenbergia peruviana A. Gray & $\mathrm{s}$ & pj & $\mathrm{Cm}$ & $\mathrm{N}$ & $\mathrm{x}$ & $4000-4400$ & Mont. 2183 & * \\
\hline \multicolumn{9}{|l|}{ Caryophyllaceae } \\
\hline Alsine cf. rupestris Muschl. & $\mathrm{h}$ & pj & $\mathrm{T}$ & $\mathrm{E}$ & $\mathrm{x}$ & 4400 & Mont. 2446b & \\
\hline Cardionema ramosissima (Weinm.) A. Nelson \& J.F. Macbr. & $\mathrm{h}$ & $\mathrm{ms}, \mathrm{pj}$ & $\mathrm{Hc}$ & $\mathrm{N}$ & $x$ & $3400-4000$ & Mont. 2544 & * \\
\hline Cerastium sp. & $\mathrm{h}$ & $\mathrm{ms}, \mathrm{pj}$ & $\mathrm{Hc}$ & - & - & $3800-4400$ & Mont. 2177 & * \\
\hline Drymaria rotundifolia A. Gray & $\mathrm{h}$ & $\mathrm{ms}, \mathrm{bf}$ & $\mathrm{Hd}$ & $\mathrm{N}$ & & $3400-3850$ & Mont. 2168a & * \\
\hline Paronychia cf. andina A. Gray & hs & pj & $\mathrm{Hc}$ & $\mathrm{N}$ & $x$ & $3850-4200$ & Mont. 2054 & \\
\hline Paronychia setigera (Gillies) F. Herm. & $\mathrm{h}$ & $\mathrm{ms}$ & $\mathrm{Hc}$ & $\mathrm{N}$ & $x$ & $3500-4000$ & Mont. 1182 & * \\
\hline Paronychia sp. & $\mathrm{h}$ & $\mathrm{ms}, \mathrm{pj}$ & $\mathrm{Cm}$ & - & - & $3800-3900$ & Mont. 2567 & \\
\hline Pycnophyllum glomeratum Mattf. & $\mathrm{h}$ & pj & $\mathrm{Cm}$ & $\mathrm{E}$ & $x$ & $4400-4600$ & Mont. 2415 & * \\
\hline Pycnophyllum molle Remy & $\mathrm{h}$ & $\mathrm{pj}, \mathrm{bf}$ & $\mathrm{Cm}$ & $\mathrm{N}$ & & $4100-4600$ & Mont. 2438 & * \\
\hline Silene andicola Gillies ex Hook. \& Arn. & $\mathrm{h}$ & $\mathrm{ms}, \mathrm{pj}$ & $\mathrm{Hc}$ & $\mathrm{N}$ & $x$ & $3600-3900$ & Mont. 2185 & * \\
\hline Silene genovevae Bocquet & $\mathrm{h}$ & $\mathrm{ms}, \mathrm{pj}$ & $\mathrm{Hc}$ & $\mathrm{N}$ & $\mathrm{x}$ & $3800-4200$ & Mont. 2268 & * \\
\hline Spergularia andina Rohrb. & $\mathrm{h}$ & $\mathrm{pj}$ & $\mathrm{Hc}$ & $\mathrm{N}$ & $\mathrm{x}$ & 4000 & $\mathrm{f} !$ & \\
\hline Spergularia fasiculata Phil. & hs & ms & $\mathrm{Nf}$ & $\mathrm{N}$ & & $3400-4200$ & Mont. 2087 & * \\
\hline Stellaria cuspidata Willd. ex Schltdl. & $\mathrm{h}$ & $\mathrm{ms}, \mathrm{pj}$ & $\mathrm{T}$ & $\mathrm{N}$ & $x$ & $3700-3850$ & Mont. 2562 & \\
\hline \multicolumn{9}{|l|}{ Convolvulaceae } \\
\hline Ipomoea minuta R. E. Fr. & $\mathrm{h}$ & $\mathrm{ms}$ & G & $\mathrm{N}$ & $\mathrm{x}$ & $3400-3500$ & Mont. 0751 & \\
\hline Ipomoea tricolor Cav. & $\mathrm{h}$ & $\mathrm{ms}$ & $\mathrm{Hc}$ & I & & $3400-3600$ & Mont. 0592 & \\
\hline \multicolumn{9}{|l|}{ Crassulaceae } \\
\hline Crassula connata (Ruiz \& Pav.) A. Berger \& al. & h, su & $\mathrm{ms}, \mathrm{pj}, \mathrm{bf}$ & $\mathrm{Hl}$ & $\mathrm{N}$ & $\mathrm{x}$ & $3500-4600$ & Mont. 1024 & * \\
\hline Echeveria peruviana Meyen & h, su & $\mathrm{ms}$ & $\mathrm{S}$ & $\mathrm{E}$ & $\mathrm{x}$ & $3400-3800$ & Mont. 0788 & \\
\hline Sedum reniforme (H. Jacobsen) Thiede \& $\mathrm{T}^{\prime}$ Hart & h, su & $\mathrm{ms}$ & $\mathrm{S}$ & $\mathrm{E}$ & $x$ & $3400-3500$ & Mont. 0753 & \\
\hline \multicolumn{9}{|l|}{ Cucurbitaceae } \\
\hline Sicyos baderoa Hook. \& Arn. & $\mathrm{h}$ & ms & Hc & $\mathrm{N}$ & & $3400-3500$ & Mont. 2154 & * \\
\hline \multicolumn{9}{|l|}{ Euphorbiaceae } \\
\hline Chamaesyce serpens (Kunth) Small & $\mathrm{h}$ & $\mathrm{ms}$ & $\mathrm{T}$ & $\mathrm{N}$ & & $3400-3900$ & Mont. 0746 & \\
\hline Euphorbia huanchahana (Klotzsch \& Garcke) Boiss. & $\mathrm{h}$ & pj & $\mathrm{Hc}$ & $\mathrm{N}$ & $\mathrm{x}$ & $4000-4100$ & Mont. 2356 & \\
\hline Euphorbia sp. & $\mathrm{h}$ & ms & Hc & $\mathrm{N}$ & & $3400-3500$ & Mont. 0603 & * \\
\hline \multicolumn{9}{|l|}{ Fabaceae } \\
\hline Adesmia miraflorensis Remy & $\mathrm{s}$ & $\mathrm{ms}, \mathrm{pj}$ & $\mathrm{Nf}$ & $\mathrm{N}$ & & $3400-3900$ & Mont. 2159 & * \\
\hline Adesmia spinosissima Meyen ex Vogel & $\mathrm{s}$ & $\mathrm{pj}$ & $\mathrm{Nf}$ & $\mathrm{N}$ & & $3600-4100$ & Mont. 0671 & \\
\hline Astragalus arequipensis Vogel & hs & $\mathrm{ms}, \mathrm{pj}, \mathrm{bf}$ & $\mathrm{Hc}$ & $\mathrm{N}$ & $\mathrm{x}$ & $3400-4400$ & Mont. 2003 & * \\
\hline Astragalus dielsii J.F. Macbr. & $\mathrm{h}$ & pj & Hc & $\mathrm{E}$ & $x$ & $4200-4400$ & Mont. 2506 & \\
\hline Astragalus micranthellus Wedd. & hs & $\mathrm{ms}, \mathrm{pj}$ & $\mathrm{Hc}$ & $\mathrm{N}$ & $x$ & $3400-3900$ & Mont. 2000 & * \\
\hline Astragalus peruvianus Vogel & $\mathrm{h}$ & $\mathrm{pj}$ & $\mathrm{Hc}$ & $\mathrm{N}$ & & $4000-4200$ & Mont. 2256 & * \\
\hline Astragalus punensis J.F. Macbr. & hs & pj & $\mathrm{Cm}$ & $\mathrm{E}$ & $x$ & $4200-4500$ & Mont. 2369 & * \\
\hline Astragalus triflorus (DC.) A. Gray & hs & pj & $\mathrm{Hc}$ & $\mathrm{N}$ & & $4000-4200$ & Mont. 0803 & \\
\hline Astragalus cf. uniflorus DC. & $\mathrm{h}$ & pj & Hc & $\mathrm{N}$ & $\mathrm{x}$ & $4000-4200$ & Mont. 2257 & * \\
\hline Astragalus sp. 1 & $\mathrm{~h}$ & pj & $\mathrm{Hc}$ & - & - & $4000-4200$ & Mont. 2192 & * \\
\hline Astragalus sp. 2 & $\mathrm{~h}$ & pj & $\mathrm{Hc}$ & - & - & 4050 & Mont. 2255 & \\
\hline Dalea cylindrica Hook. & hs & $\mathrm{ms}$ & $\mathrm{Nf}$ & $\mathrm{N}$ & & $3600-3750$ & Mont. 2163 & * \\
\hline Dalea onobrychis DC. & hs & $\mathrm{ms}$ & $\mathrm{Nf}$ & $\mathrm{N}$ & & $3500-3700$ & Mont. 0699 & * \\
\hline Lathyrus magellanicus Lam. & $\mathrm{h}$ & $\mathrm{ms}$ & Hc & $\mathrm{N}$ & $x$ & $3400-3600$ & Mont. 1232 & \\
\hline Lupinus cf. ananeanus Ulbr. & $\mathrm{h}$ & pj & $\mathrm{Hc}$ & $\mathrm{N}$ & $x$ & $4000-4200$ & Mont. 2197 & * \\
\hline Lupinus paruroensis C.P. Sm. & $\mathrm{s}$ & $\mathrm{ms}, \mathrm{pj}$ & $\mathrm{Nf}$ & $\mathrm{E}$ & & $3500-4100$ & Mont. 2100 & * \\
\hline Lupinus sp. 1 & $\mathrm{~h}$ & $\mathrm{pj}$ & Hc & - & - & $4050-4200$ & Mont. 2251 & \\
\hline Lupinus sp. 2 & $\mathrm{~h}$ & pj & $\mathrm{Hc}$ & - & - & $4450-4600$ & Mont. 2424 & \\
\hline Medicago lupulina L. & $\mathrm{h}$ & $\mathrm{ms}$ & $\mathrm{Hc}$ & I & $x$ & $3400-3700$ & Mont. 0633 & * \\
\hline Otholobium munyense (J.F. Macbr.) J.W. Grimes & $\mathrm{s}$ & ms & $\mathrm{F}$ & $\mathrm{N}$ & $x$ & 3450 & Mont. 0587 & * \\
\hline
\end{tabular}

(Continúa...) 
Apéndice 1. Continuación.

\begin{tabular}{|c|c|c|c|c|c|c|c|c|}
\hline Taxón & FC & $\mathrm{FE}$ & FB & St & NR & Altitud (m) & Voucher & \\
\hline Trifolium amabile Kunth & $\mathrm{h}$ & ms, pj & Hc & I & $\mathrm{x}$ & $3400-4400$ & Mont. 2007 & * \\
\hline Vicia cf. andicola Kunth & $\mathrm{h}$ & $\mathrm{ms}$ & $\mathrm{Hc}$ & $\mathrm{N}$ & $\mathrm{x}$ & $3600-3700$ & Mont. 0729 & * \\
\hline \multicolumn{9}{|l|}{ Gentianaceae } \\
\hline Gentiana sedifolia Kunth & $\mathrm{h}$ & $\mathrm{ms}, \mathrm{pj}$ & $\mathrm{Hl}$ & $\mathrm{N}$ & $\mathrm{x}$ & $3500-3700$ & Mont. 2288 & \\
\hline Gentiana sp. & $\mathrm{h}$ & bf & $\mathrm{Hl}$ & - & - & 3400 & Mont. 2221 & \\
\hline Gentianella poculifera (Gilg) T.N. Ho \& S.W. Liu & $\mathrm{h}$ & $\mathrm{pj}$ & $\mathrm{Hl}$ & $\mathrm{E}$ & $x$ & $4000-4200$ & Mont. 2067 & * \\
\hline Gentianella potamophila (Gilg) Zarucchi & $\mathrm{h}$ & $\mathrm{pj}, \mathrm{bf}$ & $\mathrm{Hl}$ & $\mathrm{E}$ & $\mathrm{x}$ & $3900-4500$ & Mont. 2293 & * \\
\hline Gentianella sp. 1 & $\mathrm{~h}$ & bf & $\mathrm{Hl}$ & - & - & $4450-4550$ & Mont. 2464 & \\
\hline Gentianella sp. 2 & $\mathrm{~h}$ & bf & $\mathrm{Hl}$ & - & - & 3900 & Mont. 2322 & \\
\hline Gentianella sp. 3 & $\mathrm{~h}$ & pj & $\mathrm{Hl}$ & - & - & $3800-4000$ & Mont. 0808 & \\
\hline \multicolumn{9}{|l|}{ Geraniaceae } \\
\hline Erodium cicutarium (L.) L'Hér. ex Aiton & $\mathrm{h}$ & ms & $\mathrm{T}$ & $\mathrm{N}$ & & $3400-3900$ & Mont. 0529 & * \\
\hline Geranium core-core Steud. & $\mathrm{h}$ & $\mathrm{pj}$ & $\mathrm{Hc}$ & $\mathrm{N}$ & & $4000-4100$ & Mont. 2551 & \\
\hline Geranium sessiliflorum Cav. & $\mathrm{h}$ & $\mathrm{ms}$ & $\mathrm{Hc}$ & $\mathrm{N}$ & & $3800-4300$ & Mont. 2142 & * \\
\hline Geranium cf. staffordianum R. Knuth & $\mathrm{h}$ & $\mathrm{ms}$ & $\mathrm{Hc}$ & $\mathrm{E}$ & $\mathrm{x}$ & 3600 & Mont. 0994 & \\
\hline Geranium sp. & $\mathrm{h}$ & $\mathrm{ms}$ & $\mathrm{T}$ & - & - & 3400 & Mont. 2206b & \\
\hline \multicolumn{9}{|l|}{ Grossulariaceae } \\
\hline Ribes brachybotrys (Wedd.) Jancz. & $\mathrm{s}, \mathrm{a}$ & $\mathrm{pj}$ & $\mathrm{F}$ & $\mathrm{N}$ & $\mathrm{x}$ & $3700-4200$ & Mont. 2532 & * \\
\hline \multicolumn{9}{|l|}{ Hydrophyllaceae } \\
\hline Nama dichotomum (Ruiz \& Pav.) Choisy & $\mathrm{h}$ & $\mathrm{ms}$ & $\mathrm{T}$ & $\mathrm{N}$ & & $3400-3600$ & Mont. 0978 & * \\
\hline Phacelia pinnatifida Griseb. ex Wedd. & $\mathrm{h}$ & $\mathrm{ms}$ & $\mathrm{T}$ & $\mathrm{N}$ & & $3550-3700$ & Mont. 2227 & * \\
\hline Phacelia secunda J.F. Gmel. & $\mathrm{h}$ & $\mathrm{ms}$ & $\mathrm{Hc}$ & $\mathrm{N}$ & $\mathrm{x}$ & 3800 & Mont. 2556 & \\
\hline \multicolumn{9}{|l|}{ Lamiaceae } \\
\hline Lepechinia meyenii (Walp.) Epling & $\mathrm{h}$ & $\mathrm{ms}, \mathrm{pj}$ & $\mathrm{Hc}$ & $\mathrm{N}$ & $\mathrm{x}$ & $3600-4050$ & Mont. 0544 & \\
\hline Mentha aquatica L. & $\mathrm{h}$ & $\mathrm{ms}$ & $\mathrm{Hd}$ & I & $\mathrm{x}$ & $3450-3900$ & Mont. 2324 & * \\
\hline Salvia oppositiflora Ruiz \& Pav. & $\mathrm{s}$ & $\mathrm{ms}$ & $\mathrm{Nf}$ & $\mathrm{E}$ & $\mathrm{x}$ & $3400-3800$ & Mont. 0517 & * \\
\hline Satureja boliviana (Benth.) Briq. & $\mathrm{s}$ & $\mathrm{ms}, \mathrm{pj}$ & $\mathrm{Nf}$ & $\mathrm{N}$ & & $3500-4100$ & Mont. 2241 & * \\
\hline Stachys cf. pusilla (Wedd.) Briq. & $\mathrm{h}$ & $\mathrm{ms}$ & $\mathrm{T}$ & $\mathrm{N}$ & $\mathrm{x}$ & 3400 & Mont. 0731 & \\
\hline \multicolumn{9}{|l|}{ Lentibulariaceae } \\
\hline Pinguicula involuta Ruiz \& Pav. & $\mathrm{h}$ & ms & $\mathrm{Hl}$ & $\mathrm{N}$ & $x$ & 3700 & $\mathrm{f} !$ & \\
\hline \multicolumn{9}{|l|}{ Loasaceae } \\
\hline Caiophora chuquitensis (Meyen) Urb. \& Gilg & $\mathrm{h}$ & pj & $\mathrm{Hc}$ & $\mathrm{N}$ & & $4050-4200$ & Mont. 2264 & * \\
\hline Caiophora cirsiifolia C. Presl & $\mathrm{h}$ & ms & $\mathrm{Hc}$ & $\mathrm{E}$ & $\mathrm{x}$ & $3400-3600$ & Mont. 2243 & * \\
\hline Caiophora rosulata (Wedd.) Urb. \& Gilg & $\mathrm{h}$ & pj & $\mathrm{Hc}$ & $\mathrm{N}$ & & $4000-4700$ & Mont. 2313 & * \\
\hline \multicolumn{9}{|l|}{ Loganiaceae } \\
\hline Buddleja coriacea Remy & a & ms, pj & $\mathrm{F}$ & $\mathrm{N}$ & & $3400-4200$ & Mont. 0518 & * \\
\hline \multicolumn{9}{|l|}{ Loranthaceae } \\
\hline Ligaria cuneifolia (Ruiz \& Pav.) Tiegh. & $\mathrm{s}, \mathrm{p}$ & ms & $\mathrm{Nf}$ & $\mathrm{N}$ & & $3400-3500$ & Mont. 0661 & * \\
\hline \multicolumn{9}{|l|}{ Malvaceae } \\
\hline Fuertesimalva echinata (C. Presl) Fryxel & $\mathrm{h}$ & $\mathrm{ms}$ & $\mathrm{Nf}$ & $\mathrm{N}$ & $\mathrm{x}$ & $3400-3500$ & Mont. 2207 & \\
\hline Nototriche anthemidifolia (J. Rémy) A.W. Hill & hs & pj & $\mathrm{Hc}$ & $\mathrm{N}$ & $\mathrm{x}$ & $4050-4600$ & Mont. 2269 & * \\
\hline Nototriche argentea A.W. Hill & $\mathrm{h}$ & pj & Нc & $\mathrm{N}$ & & $4400-4600$ & Mont. 2363b & \\
\hline Nototriche cf. digitulifolia Hill & hs & pj & $\mathrm{Hc}$ & $\mathrm{E}$ & $x$ & $4200-4300$ & Mont. 2595 & \\
\hline Nototriche longirostris (Wedd.) A.W. Hill & $\mathrm{h}$ & $\mathrm{pj}$ & $\mathrm{Hc}$ & $\mathrm{N}$ & $\mathrm{x}$ & $4200-4600$ & Mont. 2482 & * \\
\hline Nototriche mandoniana (Wedd.) A.W. Hill & hs & pj & $\mathrm{Hc}$ & $\mathrm{N}$ & $\mathrm{x}$ & $4000-4200$ & Mont. 2171 & * \\
\hline Nototriche pedatiloba Hill & hs & pj & $\mathrm{Hc}$ & $\mathrm{N}$ & $\mathrm{x}$ & $4400-4600$ & Mont. 2474 & \\
\hline Nototriche pedicularifolia A.W. Hill & hs & $\mathrm{pj}$ & $\mathrm{Hc}$ & $\mathrm{N}$ & $\mathrm{x}$ & $4000-4700$ & Mont. 2305 & * \\
\hline Nototriche pusilla A. W. Hill & $\mathrm{h}$ & $\mathrm{pj}$ & $\mathrm{Hc}$ & $\mathrm{N}$ & $\mathrm{x}$ & $4300-4500$ & Mont. 2518 & \\
\hline Nototriche turritella Hill & $\mathrm{h}$ & $\mathrm{pj}$ & $\mathrm{Hc}$ & $\mathrm{N}$ & $\mathrm{x}$ & $4000-4200$ & Mont. 2170 & * \\
\hline Nototriche sp. & $\mathrm{h}$ & $\mathrm{pj}$ & $\mathrm{Hc}$ & - & - & 4380 & Mont. 2447 & \\
\hline Tarasa nototrichoides (Hochr.) Krapovickas & $\mathrm{h}$ & pj & $\mathrm{Hc}$ & $\mathrm{E}$ & $\mathrm{x}$ & $4000-4300$ & Mont. 2388 & \\
\hline Tarasa tarapacana (Phil.) Krapov. & $\mathrm{h}$ & $\mathrm{ms}$ & $\mathrm{Hc}$ & $\mathrm{N}$ & $\mathrm{x}$ & $3400-4000$ & Mont. 2010 & * \\
\hline Tarasa tenuis Krapov. & $\mathrm{h}$ & $\mathrm{ms}$ & $\mathrm{Hc}$ & $\mathrm{N}$ & $\mathrm{x}$ & $3600-3750$ & Mont. 2162 & \\
\hline Tarasa urbaniana (Ulbr.) Krapov & $\mathrm{h}$ & $\mathrm{ms}$ & $\mathrm{Hc}$ & $\mathrm{N}$ & $\mathrm{x}$ & $3600-3700$ & Mont. 2166b & \\
\hline Nyctaginaceae & & & & & & & & \\
\hline Mirabilis expansa (Ruiz \& Pav.) Standl. & $\mathrm{h}$ & ms & $\mathrm{Hc}$ & $\mathrm{N}$ & $x$ & $3400-3600$ & Mont. 1017 & * \\
\hline Onagraceae & & & & & & & & \\
\hline Epilobium denticulatum Ruiz \& Pav. & $\mathrm{h}$ & $\mathrm{ms}$ & $\mathrm{T}$ & $\mathrm{N}$ & & $3400-3500$ & Mont. 0609 & \\
\hline Oenothera multicaulis Ruiz \& Pav. & $\mathrm{h}$ & $\mathrm{ms}, \mathrm{pj}$ & $\mathrm{Hc}$ & $\mathrm{N}$ & $x$ & $3500-3900$ & Mont. 2120 & * \\
\hline Oenothera nocturna Jacq. & $\mathrm{h}$ & $\mathrm{ms}$ & $\mathrm{Hc}$ & $\mathrm{N}$ & $\mathrm{x}$ & $3500-3600$ & Mont. 2094 & \\
\hline Oenothera rosea L'Hér. ex Aiton & $\mathrm{h}$ & $\mathrm{ms}$ & $\mathrm{Hl}$ & $\mathrm{N}$ & & $3400-3600$ & Mont. 0614 & \\
\hline Oenothera sp. & $\mathrm{h}$ & $\mathrm{ms}, \mathrm{pj}$ & $\mathrm{Hc}$ & - & - & $3800-4100$ & Mont. 2276 & * \\
\hline Oxalidaceae & & & & & & & & \\
\hline Oxalis calachaccensis R. Knuth & $\mathrm{h}$ & ms, pj & $\mathrm{T}$ & $\mathrm{N}$ & $\mathrm{x}$ & $3750-4350$ & Mont. 2391 & * \\
\hline Oxalis debilis Kunth & $\mathrm{h}$ & $\mathrm{ms}$ & G & $\mathrm{N}$ & $\mathrm{x}$ & $3400-3500$ & Mont. 0717 & * \\
\hline Oxalis megalorrhiza Jacq. & $\mathrm{h}$ & $\mathrm{ms}$ & G & $\mathrm{N}$ & $x$ & $3400-4100$ & Mont. 2090 & * \\
\hline Oxalis sp. & $\mathrm{h}$ & $\mathrm{ms}$ & G & - & - & 3500 & $\mathrm{f} !$ & \\
\hline Piperaceae & & & & & & & & \\
\hline Peperomia peruviana Dahlst. & $\mathrm{h}$ & $\mathrm{ms}$ & $\mathrm{T}$ & $\mathrm{N}$ & $x$ & $3400-3900$ & Mont. 2229 & \\
\hline
\end{tabular}

(Continúa...) 
Plantaginaceae

Bougueria nubicola Decne.

Plantago australis Lam. subsp. hirtella

Plantago australis Lam. subsp. pflanzii

Plantago lanceolata $\mathrm{L}$.

Plantago major $\mathrm{L}$.

Plantago monticola Decne.

Plantago myosuros Lam.

Plantago cf. nubigena Kunth

Plantago rigida Kunth

Plantago sericea Ruiz \& Pav.

Plantago sericea var. lanuginosa Griseb.

Plantago tubulosa Decne.

Polemoniaceae

Cantua buxifolia Juss. ex Lam.

Gilia laciniata Ruiz \& Pav.

Huthia sp.

Polygonaceae

Rumex crispus $\mathrm{L}$.

Portulacaceae

Calandrinia acaulis Kunth

Calandrinia ciliata (Ruiz \& Pav.) DC.

Portulaca oleracea L.

Portulaca perennis R.E. Fr.

Ranunculaceae

Rosaceae

Ranunculus flagelliformis $\mathrm{Sm}$.

Lachemilla diplophylla (Diels) Rothm.

Lachemilla pinnata (Ruiz \& Pav.) Rothm.

Polylepis besseri Hieron.

Tetraglochin cristatum (Britton) Rothm.

Rubiaceae

Galium aparine L.

Santalaceae

Galium corymbosum Ruiz \& Pav.

Quinchamalium procumbens Ruiz \& Pav.

Quinchamalium sp.

Saxifragaceae

Escallonia myrtilloides L. $\mathrm{f}$.

Scrophulariaceae

Bartsia cf. crenoloba Wedd.

Bartsia diffusa Benth.

Bartsia peruviana Walp.

Bartsia cf. weberbaueri Diels

Castilleja pumila (Benth.) Wedd.

Limosella aquatica $\mathrm{L}$.

Mimulus glabratus Kunth

Orthocarpus laciniatus (Hook. \& Arn.) D.D. Keck

Ourisia muscosa Benth.

Solanaceae

Dunalia spinosa (Meyen) Dammer

Lycianthes lycioides (L.) Hassl.

Nicotiana rustica $\mathrm{L}$.

Nicotiana undulata Ruiz \& Pav.

Salpichroa glandulosa (Hook.) Miers

Salpichroa tristis Miers

Solanum americanum Mill.

Solanum bukasovii Juz.

Solanum chamaesarachidium Bitter

Solanum excisirhombeum Bitter

Solanum nigrum $\mathrm{L}$.

Solanum nitidum Ruiz \& Pav.

Solanum pentlandii Dunal

Solanum radicans $\mathrm{L}$. $\mathrm{f}$.

Urticaceae

Urtica echinata Benth.

Urtica flabellata Kunth

\section{Valerianaceae}

Stangea cf. rhizantha (A. Gray) Killip

$\begin{array}{ccc}\text { h } & \text { ms, pj } & \text { Hc } \\ \text { h } & \text { ms } & \mathrm{Hl} \\ \text { h } & \text { pj } & \mathrm{Hc} \\ \text { h } & \text { ms } & \mathrm{Hl} \\ \text { h } & \text { ms } & \mathrm{Hl} \\ \text { h } & \text { ms, pj } & \mathrm{Hc} \\ \text { h } & \text { ms } & \mathrm{T} \\ \text { hs } & \text { pj } & \mathrm{Cm} \\ \text { hs } & \text { bf } & \mathrm{Cm} \\ \text { hs } & \text { ms, pj } & \mathrm{Cm} \\ \text { hs } & \text { pj } & \mathrm{Hc} \\ \text { h } & \text { ms, pj } & \mathrm{Hl}\end{array}$

Mont. 2145

Mont. 0520

Mont. 2277

Mont. 0599

Mont. 2131

Mont. 1006

Mont. 2004

Mont. 2023

3500-3700

3500-3700

$4400-4700$

3550-3800

3850-4300

3400-4100

Mont. 2239

Mont. 0688

Mont. 1226

3400-3800

Mont. 2349

3800-4000 Mont. 2198c

4230

Mont. 2591

$3400-3600$

Mont. 0525

Mont. 2308

3800-4200

3900-4100

3400-3600

Mont. 0952

Mont. 1015

3400-3600

Mont. 0714

$\begin{array}{llllll}\text { h pj, bf } & \text { Hd } & \text { N } & \text { x } & 4500\end{array}$

f!

h bf $\quad \mathrm{Hd}$

ms, pj $\mathrm{Hl}$

ms $\quad \mathrm{F}$

$\mathrm{ms}, \mathrm{pj}, \mathrm{bf} \quad \mathrm{Nf}$

4200-4600

Mont. 2453

Mont. 2526

Mont. 1185

3700-3900

$3700-4500$

Mont. 2529

Mont. 2111

Mont. 2033

$3400-4200$

$\begin{array}{cc}3400-4000 & \text { Mont. } 2166 \\ 3950 & \text { Mont. } 0640\end{array}$

$\begin{array}{cccccr}\text { h, p } & \text { ms, pj } & \text { Cm } & \text { N } & & 3400-4000 \\ \text { h } & \text { pj } & \text { T } & \text { N } & \text { x } & 3950\end{array}$

Mont. 0640

$3400-4200$

Mont. 2485

Mont. 2497

$3800-4200$

$4300-4700$

3400-4000

4500-4700

3600-4200

4200-4400

3700-4100

3900-4000

3800-4400

Mont. 0939

Mont. 2024

Mont. 2436

Mont. 2062

Mont. 2590

Mont. 1013

Mont. 2550

pj Hc

pj $\quad \mathrm{Hl}$

$\mathrm{N}$

f!

3400-3500

3400-3600

Mont. 0580

Mont. 0596

Mont. 2283

$\begin{array}{cc}3500-3800 & \text { Mont. } 2283 \\ 3700 & \text { Mont. } 2021\end{array}$

3500-4200

3400-3500

Mont. 2549

Mont. 0689

$3400-3600 \mathrm{~m}$

3600-4100

Mont. 0516

Mont. 0680

3900

3400-3500

Mont. 2144

Mont. 0920a

3400-3500 Mont. 0920b

3400-3500

Mont. $0920 \mathrm{~b}$

Mont. 2620

$\begin{array}{lccccc}\text { hs } & \text { pj } & \text { Hc } & \text { N } & \text { x } & 3900-4000 \\ \text { hs } & \text { ms } & \text { Hc } & \text { N } & & 3400\end{array}$

Mont. 1220

Mont. 2265

3400-4500

Mont. 1027

4300

Mont. 2446

(Continúa...) 
Apéndice 1. Continuación

\begin{tabular}{|c|c|c|c|c|c|c|c|c|}
\hline Taxón & FC & FE & FB & St & NR & Altitud (m) & Voucher & \\
\hline Valeriana coarctata Ruiz \& Pav. & $\mathrm{h}$ & pj & $\mathrm{Hc}$ & $\mathrm{E}$ & $\mathrm{x}$ & $3800-3900$ & Mont. 2559 & \\
\hline Valeriana cf. decussata Ruiz \& Pav. & $\mathrm{h}$ & pj & $\mathrm{Hc}$ & $\mathrm{N}$ & $\mathrm{x}$ & $3600-3800$ & Mont. 2002b & \\
\hline Valeriana interrupta Ruiz \& Pav. & $\mathrm{h}$ & $\mathrm{ms}, \mathrm{pj}$ & $\mathrm{Hc}$ & $\mathrm{N}$ & $x$ & $3500-4100$ & Mont. 0990 & * \\
\hline Valeriana nivalis Wedd. & hs & $\mathrm{pj}$ & $\mathrm{Hc}$ & $\mathrm{N}$ & & $4000-4300$ & Mont. 2248 & * \\
\hline Valeriana cf. radicata Graebn. & $\mathrm{h}$ & $\mathrm{ms}$ & $\mathrm{Hc}$ & $\mathrm{E}$ & $x$ & $3700-3800$ & Mont. 2036 & \\
\hline Valeriana sp. & $\mathrm{h}$ & pj & $\mathrm{Hc}$ & - & - & $4000-4200$ & $\mathrm{f} !$ & \\
\hline \multicolumn{9}{|l|}{ Verbenaceae } \\
\hline Aloysia citriodora Paláu & $\mathrm{s}$ & $\mathrm{ms}$ & $\mathrm{F}$ & I & $x$ & $3500-3700$ & Mont. 2486 & * \\
\hline Junellia arequipense (Botta) Botta & $\mathrm{s}$ & $\mathrm{ms}$ & $\mathrm{Nf}$ & $\mathrm{E}$ & $x$ & $3400-3600$ & Mont. 2215 & * \\
\hline Junellia minima (Meyen) Moldenke & $\mathrm{s}$ & pj & $\mathrm{Cm}$ & $\mathrm{N}$ & & $4300-4500$ & Mont. 2576 & \\
\hline Verbena hispida Ruiz \& Pav. & $\mathrm{h}$ & $\mathrm{ms}$ & $\mathrm{Hc}$ & $\mathrm{N}$ & & $3500-3700$ & Mont. 0752 & \\
\hline \multicolumn{9}{|l|}{ Violaceae } \\
\hline Viola granulosa Wedd. & $\mathrm{h}$ & pj & $\mathrm{Hc}$ & $\mathrm{N}$ & $\mathrm{x}$ & $4100-4200$ & Mont. 2297 & \\
\hline Viola hillii W. Becker & $\mathrm{h}$ & pj & $\mathrm{Hc}$ & $\mathrm{N}$ & $x$ & $4200-4500$ & Mont. 2572 & * \\
\hline Viola sp. & $\mathrm{h}$ & pj & $\mathrm{Hc}$ & - & - & 4150 & Mont. 2298 & \\
\hline
\end{tabular}

